\title{
Three-Echelon Inventory Model with Permissible Delay in Payments under Controllable Lead Time and Backorder Consideration
}

\author{
M. F. Yang and Wei-Chung Tseng \\ Department of Transportation Science, National Taiwan Ocean University, Keelung City 202, Taiwan
}

Correspondence should be addressed to M. F. Yang; yang60429@mail.ntou.edu.tw

Received 25 January 2014; Accepted 19 March 2014; Published 30 April 2014

Academic Editor: Sri Sridharan

Copyright (C) 2014 M. F. Yang and W.-C. Tseng. This is an open access article distributed under the Creative Commons Attribution License, which permits unrestricted use, distribution, and reproduction in any medium, provided the original work is properly cited.

\begin{abstract}
This paper proposes a three-echelon inventory model with permissible delay in payments under controllable lead time and backorder consideration to find out the suitable inventory policy to enhance profit of the supply chain. In today's highly competitive market, the supply chain management has become a critical issue in both practice and academic and supply chain members have to cooperate with each other to bring more benefits. In addition, the inventory policy is a key factor to influence the performance of the supply chain. Therefore, in this paper, we develop a three-echelon inventory model with permissible delay in payments under controllable lead time and backorder consideration. Furthermore, the purpose of this paper is to maximize the joint expect total profit on inventory model and attempt to discuss the inventory policy under different conditions. Finally, with a numerical example provided here to illustrate the solution procedure, we may discover that decision-makers can control lead time and payment time to enhance the performance of the supply chain.
\end{abstract}

\section{Introduction}

Inventory policy and supply chain are closely linked to each other; inventory policy can easily influence the performance of supply chain [1]. Jammernegg and Reiner [2] pointed out that the efficient inventory management can enhance performance of supply chain. To enhance the performance of the supply chain, firms play the different role in the supply chain, and they should have a good partnership and share the information with each other. Cachon and Fisher [3] established a platform for firms in the supply chain; it could share information, built the forecast model, and reduced the risk from inventory model. Olson and Xie [4] proposed that vendor and buyer should use the same inventory system to cooperate with each other, and inappropriate inventory policy might cause firms to bear great loss. With the above discussion, the proper inventory policy is a critical part in the supply chain. We would like to develop an integrated inventory model to obtain the optimal solutions and attempt to discuss the inventory policy, and next we have to review some previous literatures to define the research gaps.

Stock is a major part in inventory policy. Excess stocks cause higher cost, and shortage cannot satisfy customer immediately. Frankel [5] believed that how to control stocks was a vital practice in supply chain. Das Roy et al. [6] extended Maddah and Jaber's [7] model to discuss an optimal shipment strategy for defective items in shortage situation. Sana [8] researched two-warehouse systems in EOQ model with limited capacity of own warehouse. Due to the stochastic demand, they regarded lost sale as penalty in shortage conditions. Nasiri et al. [9] emphasized that firms should keep safety stocks to satisfy customer under instable demands; hence, how to keep adequate stocks is an extremely critical issue in inventory policy. Shortage often causes a great sales loss, normally; firms adopt backorder method to lower sales loss; backorder means the buyer does not receive cargos in time and allows cargos delivery late. Lo et al. [10] indicated that backorder was an important factor in their inventory 
model; Pan et al. [11] added backorder into their inventory model that considered lead time and safety factor. When shortage occurs, in addition to using backorder method to handle unsatisfied customers, firms can also offer few discounts to save customers. Ouyang et al. [12] discussed the impact of backorder discount in the inventory model under backorder consideration. Pan and Hsiao [13] established an integrated inventory model with controllable lead time and backorder discount considerations. Therefore, backorder consideration should be mentioned in inventory model.

In addition to backorder method, compressed lead time can also alleviate shortage condition. Lead time includes preparative time for send orders, set-up time of facilities, productive time, transportation time, and so on. When the firms face the uncertain demands, they usually compress the lead time. Chandra and Grabis [14] indicated that short lead time could enhance the service level and lower inventory level effectively, but short lead time also caused highly order cost. The previous researches have regarded lead time as a constant, but more and more researches have regarded lead time as a variable or controllable parameter in recent years; BenDaya and Raouf [15] added lead time as a decision variable in the inventory model. Xia and Huang [16] established an inventory model with quantity discount and controllable lead time when demand distribution was unknown. And Song et al. [17] studied the effects of controllable lead time in the inventory model; they indicated that shorter lead time can not only satisfy customer's demand, but also lower inventory level effectively. In 2008, Lo and Yang [18] proposed a notable idea. They showed the relationship between lead time and delay payment time, and their cost functions in the model would be divided into two cases by different payment times. But they considered lead time as a constant. If the lead time is regarded as a variable, it is interesting to know whether we can find out the better inventory policy by controlling lead time and payment time.

Permissible delay in payments is a common commercial strategy in real life; the purpose of this strategy is to enhance elasticity of capital. In other words, firms can obtain additional interest income from unpaid payments, but equally firms may pay the additional opportunity cost in some conditions. Lo and Yang [18] indicated that firms should let payment time be longer than lead time to obtain additional interest income. Chen and Kang [19] established an integrated inventory model with permissible delay in payments. Zhu et al. [20] considered permissible delay in payments in their inventory model with limited storage capacity and shortage. Huang et al. [21] proposed an inventory policy between buyer and vendor and studied the relationship between permissible delay in payments and order-processing time reduction. Sana and Chaudhuri [22] proposed a deterministic EOQ model with delays in payments and price-discount offers; they considered various types of deterministic demands for retailer to operate their model. They all have added delay payment method in their inventory models. To fit the real life, inventory model should take delay payment into account.

There are many issues that can be discussed in inventory model, and we reviewed the literatures about backorder, controllable lead time, and permissible delay in payment. Shortage is an extremely common condition in real life and always accompanies sales loss. Hence, in the second paragraph, we simply defined backorder. To lower the sales loss, the backorder method is widely used in years. After reviewing Ouyang et al. [12] and Pan and Hsiao's [13] research, we would like to regard backorder as a major condition in our inventory model.

In the third paragraph, we knew that compressed lead time was another method to alleviate shortage condition. Recently researches have indicated that the controllable lead time is a popular issue in inventory model. Lead time consists of many components, and these components such as productive process and transportation process can be compressed by renewing facilities or using fast vehicles. Short lead time can respond to demand quickly and enhance the service level. With high service level, firms can gain more revenue from their customers but compressing lead time will cause additional cost to firms. With inspiration from Lo and Yang's [18] research, it is interesting to know whether we can find out better inventory policy by controlling lead time and payment time.

In the fourth paragraph, we discussed the permissible delay in payment, a common commercial strategy in business; the strategy aims to enhance the elasticity of capital. Vendor always benefit from interest income by delaying payment time, but buyer would pay an additional opportunity cost. Generally, supply chain can benefit from delay strategy; hence, how to maximize the total profit by adjusting payment time is a potential issue to discuss.

To simulate supply chain completely, we would like to discuss the above-mentioned ideas in three-echelon inventory model. There are several researches that have studied inventory problems in three-echelon models. Roy et al. [23] built a three-echelon supply chain model involving newsboy problem to deal with the inventory control problems. They also claimed that uncertain demand may cause shortage condition in their integrated inventory model. Sana [24] investigated an EOQ/EPQ model in three-echelon supply chain management; he focused on the volume flexibility and replenishment lot size problem in a collaborating system. Pal et al. [25] established a three-echelon inventory model; they considered multi-items and multisuppliers to make model more realistic. Sana et al. [26] proposed a three-echelon supply chain model with multiple suppliers, manufacturers, and retailers for multiple items. They aimed to compare the expected profit between the collaborating system and Stakelberg game structure. Three-echelon inventory model has clearly shown how supply chain works in the above researches.

With the above discussion, we would like to combine the concepts of Pan and Hsiao [13] and Lo and Yang's [18] researches to develop a three-echelon integrated inventory model with delay payment, controllable lead time, and backorder consideration. In this paper, we can determine our proposed inventory model's optimal solutions and sensitivity analysis of decision variable. Finally, with appropriate backorder condition, we can attempt to determine better inventory policy by controlling lead time and payment time. 


\section{Notations and Assumptions}

Tersine [27] believed that lead time was the time between the placement of an order and delivery of cargos. And lead time can be divided into preparative time for send orders, set-up time of facilities, productive time of products, transportation time, and so on. Lead time always includes transportation and productive process, but supplier just supplies the raw materials to the manufacturer. Hence we may suppose that manufacturer's lead time only includes transportation process, and retailer's lead time includes both. Lead time can be reduced by paying additional cost, and this additional cost was called "crashing cost" $[12,20,21]$. According to Pan and Hsiao's [13] research, retailer's lead time consisted of $n$ mutually independent components; every component has a normal duration $T_{j}$ and a minimum duration $t_{j}, j=$ $1,2, \ldots, n$, given $C(L)=C_{j}\left(L_{r j-1}-L_{r}\right)+\sum_{k=1}^{j-1} C_{j}\left(T_{j}-t_{j}\right)$ for $L_{r} \in\left[L_{r j}, L_{r j-1}\right]$ to compute the crashing cost. To establish the inventory model, the following notations and assumptions are used.

\subsection{Notations}

$L_{r}$ : the length of lead time of retailer, a decision variable

$Q_{i}$ : economic order quantity of the $i$ th model, a decision variable $(i=1,2,3,4)$

$\pi_{x}$ : backorder discount per unit during the short-age period, a decision variable.

$D$ : average demand per year

$P$ : production rate of manufacturer

$D_{r}$ : demand rate of retailer

$X$ : manufacturer's permissible delay period

$Y$ : retailer's permissible delay period

$k$ : the safety factor of the inventory

$q$ : the probability of shortage during an order period

$\beta$ : the proportion of permissible backorder during the shortage period

$\beta_{0}$ : the upper bound of the backorder ratio

$\pi_{0}$ : marginal profit per unit

$x$ : the random demand during the lead time

$\mu$ : the average of the daily demand during the lead time

$\sigma$ : the standard deviation of the daily demand during the lead time

$C_{s}$ : supplier's purchasing cost per unit

$A_{s}$ : supplier's ordering cost per order

$W_{s}$ : supplier's inspecting cost per unit

$C_{r s}:$ supplier's repair cost per unit

$Z_{s}$ : the probability of imperfect products from supplier $h_{s}$ : supplier's holding cost per unit per production run

$I_{s p}$ : supplier's capital opportunity cost per dollar per year

$I_{s e}$ : supplier's interest earned per dollar per year

$C_{m}$ : manufacturer's purchasing cost per unit

$A_{m}$ : manufacturer's ordering cost per order

$W_{m}$ : manufacturer's inspecting cost per unit

$C_{r m}$ : manufacturer's repair cost per unit

$Z_{m}$ : the probability of imperfect products from manufacturer

$F_{m}$ : manufacturer's transportation cost per order

$h_{m}$ : manufacturer's holding cost per unit per production run

$L_{m}$ : the length of lead time of manufacturer

$I_{m p}$ : manufacturer's capital opportunity cost per dollar per year

$I_{m e}:$ manufacturer's interest earned per dollar per year

$n_{m}$ : the number of shipments per production run from manufacturer to retailer

$C_{r}$ : retailer's purchasing cost per unit

$p$ : retailer's selling price per unit

$A_{r}$ : retailer's ordering cost per order

$F_{r}$ : retailer's transportation cost per order

$h_{r}$ : retailer's holding cost per unit per production run

$I_{r p}$ : retailer's capital opportunity cost per dollar per year

$I_{r e}$ : retailer's interest earned per dollar per year

$n_{r}$ : the number of shipments per production run from retailer to customer

$C_{j}$ : the crashing cost at the $j$ th component in $L_{r}(j=$ $1,2)$

$T_{j}$ : the $j$ th component in normal duration $(j=1,2)$

$t_{j}$ : the $j$ th component in minimum duration $(j=1,2)$

$\emptyset(k)$ : probability density function

$\theta(k):$ probability distribution function

$C(L)$ : the total crashing cost per production run

$\mathrm{TP}_{s}$ : supplier's total annual profit

$\mathrm{TP}_{m}$ : manufacturer's total annual profit

$\mathrm{TP}_{r}$ : retailer's total annual profit

JTP: the joint total annual profit

EJTP: the expected joint total annual profit. 


\subsection{Assumptions}

(1) This is a single supplier, a single manufacturer, and a single retailer for a single product.

(2) Shortages are not allowed at supplier and manufacturer.

(3) Lead time of manufacturer includes transportation process, and lead time of retailer includes transportation and productive process.

(4) The lead time of retailer consists of $n$ mutually independent components; the $j$ th components have a normal duration $T_{j}$ and a minimum duration $t_{j}$, $j=1,2, \ldots, n$.

(5) Let $L_{r j}$ be the length of lead time with components $1,2, \ldots, j$ crashed to their minimum values, and $L_{r j}$ can be expressed as $L_{r j}=\sum_{k=1}^{n} T_{j}-\sum_{k=1}^{j}\left(T_{j}-t_{j}\right)$, for $j=1,2, \ldots, n$; hence, with a given lead time $L_{r} \in\left[L_{r j}, L_{r j-1}\right]$, the crashing cost per production run is given by $C(L)=C_{j}\left(L_{r j-1}-L_{r}\right)+\sum_{k=1}^{j-1} C_{j}\left(T_{j}-t_{j}\right)$ for $L_{r} \in\left[L_{r j}, L_{r j-1}\right]$.

(6) The relationship between the supplier's purchasing $\operatorname{cost} C_{s}$, manufacturer's purchasing cost $C_{m}$, retailer's purchasing cost $C_{r}$, and retailer's selling price $p$ is $p \geq C_{r} \geq C_{m} \geq C_{s}$.

(7) We regard imperfect production as basic condition in our proposed model, and inspecting costs are equal to $W_{s} D$ and $W_{m} D$ and repair costs are equal to $C_{r s} Z_{s} D$ and $C_{r m} Z_{m} D$ [18].

(8) The imperfect products will completely and immediately fix [18].

(9) The supplier offers the manufacturer a permissible delay period $X$. During this permissible delay period, the manufacturer sells the goods and uses the sale revenue to earn interest at a rate of $I_{m e}$. At the end of this time period, the manufacturer pays the purchasing cost to the supplier and the rest of the goods in the warehouse bring a capital opportunity cost at a rate of $I_{m p}$.

(10) The time horizon is infinite.

(11) Lead time is deterministic and lead time demand $x$ has finite mean $u L$ and variance $\sigma_{L}^{2}=\sigma^{2} L$ [13].

(12) The reorder point $=u L+k \sigma_{L}$, where $k$ is a safety point [13].

(13) Inventory is continuously reviewed and replenishments are made whenever the inventory level falls to the reorder point $r$.

\section{Building Model}

In this section, we discuss and develop the supplier's model, manufacture's model, and retailer's model in following section and combine the above models to an integrated inventory model. To build the proposed model, we extended Lo and Yang's [18] research to compute relevant opportunity cost and interest income and referred to Pan and Hsiao's [13] research to compute relevant shortage and crashing cost. As for the holding cost, it could be computed by using integration in Appendix A's Figures 1, 2, and 3 [28].

3.1. The Supplier's Total Annual Profit. In each production run, the supplier's costs include ordering cost, inspecting cost, repair cost, holding cost, and opportunity cost. And the supplier's revenues include sales revenue and interest income. With permissible delay in payments, we knew that, if payment time was longer than lead time, then it would bring additional interest income to the manufacturer; in addition, the supplier must pay equally opportunity cost. If payment time was shorter than lead time, then it would bring additional opportunity cost and fewer interest income to manufacturer; also, the supplier could earn interest income and pay fewer opportunity cost. Owing to the fact that the supplier's profit function has that two following cases, based on length of lead time $L_{m}$ and payment time $X$, the different parts between two possible cases are as follows.

Case 1. If $L_{m}<X$, then

(i) transfer opportunity cost $=C_{s} I_{s p}\left(Q_{i} X-Q_{i} / 2\right)$.

Case 2. If $L_{m} \geq X$, then

(i) transfer opportunity cost $=C_{s} I_{s p}\left(Q_{i} X\right)^{2} / 2 Q_{i}$;

(ii) transfer interest income $=C_{m} I_{s e}\left(Q_{i}-Q_{i} X\right)^{2} / 2 Q_{i}$.

Thus, $\mathrm{TP}_{s 1}$ and $\mathrm{TP}_{s 2}$ are given by

$\mathrm{TP}_{s 1}=$ sales revenue - ordering cost - inspecting cost

- repair cost - holding cost - opportunity cost

$$
\begin{aligned}
= & D\left(C_{m}-C_{s}\right)-\frac{A_{s} D}{Q_{i}}-W_{s} D-C_{r s} Z_{s} D \\
& -D h_{s}\left[\left(\frac{1}{2}-Z_{s}^{2}\right) \frac{Q_{i}}{P}\right]-C_{s} I_{s p}\left(Q_{i} X-\frac{Q_{i}}{2}\right),
\end{aligned}
$$

$\mathrm{TP}_{s 2}=$ sales revenue - ordering cost

- inspecting cost - repair cost - holding cost

- opportunity cost + interest income

$$
\begin{gathered}
=D\left(C_{m}-C_{s}\right)-\frac{A_{s} D}{Q_{i}}-W_{s} D-C_{r s} Z_{s} D \\
-D h_{s}\left[\left(\frac{1}{2}-Z_{s}^{2}\right) \frac{Q_{i}}{P}\right]-\frac{C_{s} I_{s p}\left(Q_{i} X\right)^{2}}{2 Q_{i}} \\
+\frac{C_{m} I_{s e}\left(Q_{i}-Q_{i} X\right)^{2}}{2 Q_{i}} .
\end{gathered}
$$

3.2. The Manufacturer's Total Annual Profit. In each production run, the manufacturer's costs include ordering cost, inspecting cost, repair cost, holding cost, transportation 


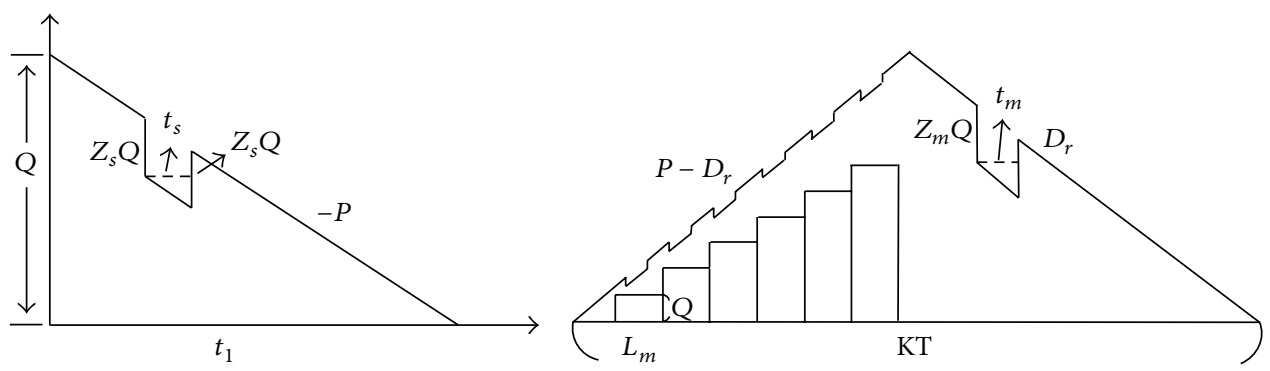

FIGURE 1: Supplier and manufacture's inventory level.

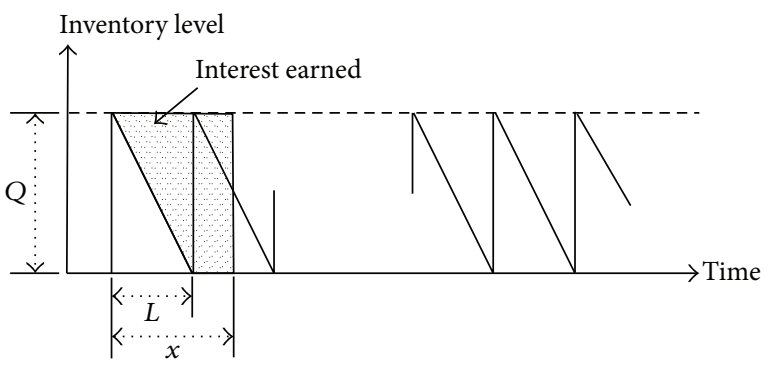

Figure 2: $L<X$.

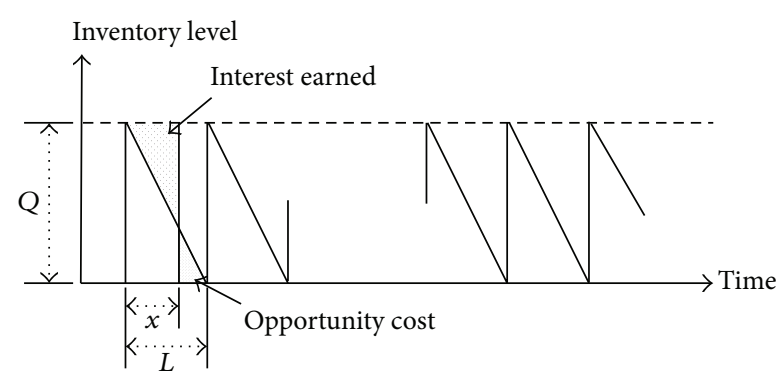

Figure 3: $L \geq X$.

cost, and opportunity cost. And the manufacturer's revenues include sales revenue and interest income. We have already known the relationship between length of lead time and payment time from Section 3.1. Owing to the fact that the manufacturer's profit function has the two following cases, based on length of lead time $L_{m}$ and payment time $X$, the different parts between two possible cases are as follows.

Case 1. If $L_{m}<X$, then

(i) interest income $=C_{r} I_{m e}\left(Q_{i} X-Q_{i} / 2\right)$;

(ii) transfer opportunity cost $=C_{m} I_{m p}\left(Q_{i} Y-Q_{i} / 2\right)$.

Case 2. If $L_{m} \geq X$, then

(i) transfer opportunity cost $=C_{m} I_{m p}\left(Q_{i} Y\right)^{2} / 2 Q_{i}$.

(ii) opportunity cost $=C_{m} I_{m p}\left(Q_{i}-Q_{i} X\right)^{2} / 2 Q_{i}$.

(iii) transfer interest income $=C_{r} I_{m e}\left(Q_{i}-Q_{i} Y\right)^{2} / 2 Q_{i}$.

(iv) interest income $=C_{r} I_{m e}\left(Q_{i} X\right)^{2} / 2 Q_{i}$.
Thus, $\mathrm{TP}_{m 1}$ and $\mathrm{TP}_{m 2}$ are given by

$\mathrm{TP}_{m 1}=$ sales revenue - ordering cost - inspecting cost

- repair cost - transportation cost - holding cost

- opportunity cost + interest income

$=D\left(C_{r}-C_{m}\right)-\frac{A_{m} D}{Q_{i}}-W_{m} D-C_{r m} Z_{m}$

$$
\begin{aligned}
& -\frac{F_{m} D}{Q_{i}}-D h_{m}\left(\left(\frac{Q_{i}}{2 D_{r}}\right)-\frac{Z_{m}^{2} Q_{i}}{D_{r}}-\frac{L_{m} n_{m}}{2}\right) \\
& +C_{r} I_{m e}\left(Q_{i} X-\frac{Q_{i}}{2}\right)-C_{m} I_{m p}\left(Q_{i} Y-\frac{Q_{i}}{2}\right),
\end{aligned}
$$

$\mathrm{TP}_{m 2}=$ sales revenue - ordering cost - inspecting cost

- repair cost - transportation cost - holding cost

- total opportunity cost + total interest income

$$
\begin{gathered}
=D\left(C_{r}-C_{m}\right)-\frac{A_{m} D}{Q_{i}}-W_{m} D-C_{r m} Z_{m} D \\
-\frac{F_{m} D}{Q_{i}}-D h_{m}\left(\left(\frac{Q_{i}}{2 D_{r}}\right)-\frac{Z_{m}^{2} Q_{i}}{D_{r}}-\frac{L_{m} n_{m}}{2}\right) \\
-\frac{C_{m} I_{m p}\left(Q_{i}-Q_{i} X\right)^{2}}{2 Q_{i}}-\frac{C_{m} I_{m p}\left(Q_{i} Y\right)^{2}}{2 Q_{i}} \\
+\frac{C_{r} I_{m e}\left(Q_{i} X\right)^{2}}{2 Q_{i}}+\frac{C_{r} I_{m e}\left(Q_{i}-Q_{i} Y\right)^{2}}{2 Q_{i}} .
\end{gathered}
$$

3.3. The Retailer's Total Annual Profit. In each production run, the retailer's costs include ordering cost, shortage cost, holding cost, transportation cost, crashing cost, and opportunity cost. And the retailer's revenues include sales revenue and interest income. We have already known the relationship between length of lead time and payment time from Section 3.1. Owing to the fact that the retailer's profit function has the two following cases, based on length of lead time $L_{r}$ and payment time $Y$, the different parts between two possible cases are as follows. 
Case 1. If $L_{r}<Y$, then

(i) interest income $=p I_{r e}\left(Q_{i} Y-Q_{i} / 2\right)$.

Case 2. If $L_{r} \geq Y$, then

(i) opportunity cost $=C_{r} I_{r p}\left(Q_{i}-Q_{i} Y\right)^{2} / 2 Q_{i}$;

(ii) interest income $=p I_{r e}\left(Q_{i} Y\right)^{2} / 2 Q_{i}$.

Thus, $\mathrm{TP}_{r 1}$ and $\mathrm{TP}_{r 2}$ are given by

$\mathrm{TP}_{r 1}=$ sales revenue - ordering cost - transportation cost

- holding cost - shortage cost

- crashing cost + interest income

$=D\left(p-C_{r}\right)-\frac{A_{r} D}{Q_{i}}-\frac{F_{r} D}{Q_{i}}$

$-\frac{1}{2} D h_{r} L_{r}\left(\frac{1}{n r}-\frac{\left(\sigma \sqrt{L_{r}} \varphi(k) n_{r} q\right)}{Q_{i}}\right)$

$-\frac{D}{Q_{i}}\left(\frac{\beta_{0}}{\pi_{0}} \pi_{x}^{2}+\pi_{0}-\beta_{0} \pi_{x}\right) \sigma \sqrt{L_{r}} \varphi(k)$

$-\frac{D}{Q_{i}}\left[C_{i}\left(L_{r i-1}-L_{r}\right)+\sum_{j=1}^{i-1} C_{j}\left(T_{j}-t_{j}\right)\right]$

$-\frac{D}{Q_{i}}\left[C_{i}\left(L_{r i-1}-L_{r}\right)+\sum_{j=1}^{i-1} C_{j}\left(T_{j}-t_{j}\right)\right]$

$+p I_{r e}\left(Q_{i} Y-\frac{Q_{i}}{2}\right)$

$\mathrm{TP}_{r 2}=$ sales revenue - ordering cost - transportation cost

- holding cost - shortage cost - crashing cost

+ interest income - opportunity cost

$=D\left(p-C_{r}\right)-\frac{A_{r} D}{Q_{i}}-\frac{F_{r} D}{Q_{i}}$

$-\frac{1}{2} D h_{r} L_{r}\left(\frac{1}{n r}-\frac{\left(\sigma \sqrt{L_{r}} \varphi(k) n_{r} q\right)}{Q_{i}}\right)$

$-\frac{D}{Q_{i}}\left(\frac{\beta_{0}}{\pi_{0}} \pi_{x}^{2}+\pi_{0}-\beta_{0} \pi_{x}\right) \sigma \sqrt{L_{r}} \varphi(k)$

$-\frac{D}{Q_{i}}\left[C_{i}\left(L_{r i-1}-L_{r}\right)+\sum_{j=1}^{i-1} C_{j}\left(T_{j}-t_{j}\right)\right]$

$-\frac{C_{r} I_{r p}\left(Q_{i}-Q_{i} Y\right)^{2}}{2 Q_{i}}+\frac{p I_{r e}\left(Q_{i} Y\right)^{2}}{2 Q_{i}}$.
3.4. The Expected Joint Total Annual Profit. Hence, the expected joint total annual profit function, $\operatorname{EJTP}\left(Q_{i}, L_{r}, \pi_{x}\right)$, can be expressed as

$\operatorname{EJTP}\left(Q_{i}, L_{r}, \pi_{x}\right)$

$= \begin{cases}\operatorname{EJTP}_{1}\left(Q_{1}, L_{r}, \pi_{x}\right)=\mathrm{TP}_{s 1}+\mathrm{TP}_{m 1}+\mathrm{TP}_{r 1} & \text { if } L_{m}<X, L_{r}<Y \\ \operatorname{EJTP}_{2}\left(Q_{2}, L_{r}, \pi_{x}\right)=\mathrm{TP}_{s 1}+\mathrm{TP}_{m 1}+\mathrm{TP}_{r 2} & \text { if } L_{m}<X, L_{r} \geq Y \\ \operatorname{EJTP}_{3}\left(Q_{3}, L_{r}, \pi_{x}\right)=\mathrm{TP}_{s 2}+\mathrm{TP}_{m 2}+\mathrm{TP}_{r 1} & \text { if } L_{m} \geq X, L_{r}<Y \\ \operatorname{EJTP}_{4}\left(Q_{4}, L_{r}, \pi_{x}\right)=\mathrm{TP}_{s 2}+\mathrm{TP}_{m 2}+\mathrm{TP}_{r 2} & \text { if } L_{m} \geq X, L_{r} \geq Y,\end{cases}$

where

$$
\begin{aligned}
& \operatorname{EJTP}_{1}\left(Q_{1}, L_{r}, \pi_{x}\right) \\
& =D\left(p-C_{s}-W_{s}-C_{r s} Z_{s}-W_{m}-C_{r m} Z_{m}\right) \\
& -\frac{1}{2} D h_{r} L_{r}\left(\frac{1}{n r}-\frac{\left(\sigma \sqrt{L_{r}} \varphi(k) n_{r} q\right)}{Q_{1}}\right) \\
& -\frac{D}{Q_{1}}\left\{A_{s}+A_{m}+F_{m}+A_{r}+F_{r}\right. \\
& +\left[\frac{\beta_{0}}{\pi_{0}} \pi_{x}^{2}+\pi_{0}-\beta_{0} \pi_{x}\right] \\
& \left.\times \sigma \sqrt{L_{r}} \varphi(k)+C(L)\right\} \\
& -D h_{s}\left[\left(\frac{1}{2}-Z_{s}^{2}\right) \frac{Q_{1}}{P}\right] \\
& -D h_{m}\left(\left(\frac{Q_{1}}{2 D_{r}}\right)-\frac{Z_{m}^{2} Q_{1}}{D_{r}}-\frac{L_{m} n_{m}}{2}\right) \\
& +\left(Q_{1} X-\frac{Q_{1}}{2}\right)\left(C_{r} I_{m e}-C_{s} I_{s p}\right) \\
& +\left(Q_{1} Y-\frac{Q_{1}}{2}\right)\left(p I_{r e}-C_{m} I_{m p}\right), \\
& \operatorname{EJTP}_{2}\left(Q_{2}, L_{r}, \pi_{x}\right) \\
& =D\left(p-C_{s}-W_{s}-C_{r s} Z_{s}-W_{m}-C_{r m} Z_{m}\right) \\
& -\frac{1}{2} D h_{r} L_{r}\left(\frac{1}{n r}-\frac{\left(\sigma \sqrt{L_{r}} \varphi(k) n_{r} q\right)}{Q_{2}}\right) \\
& -\frac{D}{Q_{2}}\left\{A_{s}+A_{m}+F_{m}+A_{r}+F_{r}\right. \\
& +\left[\frac{\beta_{0}}{\pi_{0}} \pi_{x}^{2}+\pi_{0}-\beta_{0} \pi_{x}\right] \\
& \left.\times \sigma \sqrt{L_{r}} \varphi(k)+C(L)\right\} \\
& -D h_{s}\left[\left(\frac{1}{2}-Z_{s}^{2}\right) \frac{Q_{2}}{P}\right] \\
& -D h_{m}\left(\left(\frac{Q_{2}}{2 D_{r}}\right)-\frac{Z_{m}^{2} Q_{2}}{D_{r}}-\frac{L_{m} n_{m}}{2}\right)
\end{aligned}
$$




$$
\begin{aligned}
& +\left(Q_{2} X-\frac{Q_{2}}{2}\right)\left(C_{m} I_{m e}-C_{s} I_{s p}\right) \\
& +\frac{\left(Q_{2} Y\right)^{2}}{2 Q_{2}}\left(C_{r} I_{m e}+p I_{r e}-C_{m} I_{m p}-C_{r} I_{r p}\right), \\
& \operatorname{EJTP}_{3}\left(Q_{3}, L_{r}, \pi_{x}\right) \\
& =D\left(p-C_{s}-W_{s}-C_{r s} Z_{s}-W_{m}-C_{r m} Z_{m}\right) \\
& -\frac{1}{2} D h_{r} L_{r}\left(\frac{1}{n r}-\frac{\left(\sigma \sqrt{L_{r}} \varphi(k) n_{r} q\right)}{Q_{3}}\right) \\
& -\frac{D}{Q_{3}}\left\{A_{s}+A_{m}+F_{m}+A_{r}+F_{r}\right. \\
& +\left[\frac{\beta_{0}}{\pi_{0}} \pi_{x}^{2}+\pi_{0}-\beta_{0} \pi_{x}\right] \\
& \left.\times \sigma \sqrt{L_{r}} \varphi(k)+C(L)\right\} \\
& -D h_{s}\left[\left(\frac{1}{2}-Z_{s}^{2}\right) \frac{Q_{3}}{P}\right] \\
& -D h_{m}\left(\left(\frac{Q_{3}}{2 D_{r}}\right)-\frac{Z_{m}^{2} Q_{3}}{D_{r}}-\frac{L_{m} n_{m}}{2}\right) \\
& +\frac{\left(Q_{3} X\right)^{2}}{2 Q_{3}}\left(C_{r} I_{m e}-C_{s} I_{s p}\right) \\
& +\frac{\left(Q_{3}-Q_{3} X\right)^{2}}{2 Q_{3}}\left(C_{m} I_{s e}-C_{m} I_{m p}\right) \\
& +\left(Q_{3} Y-\frac{Q_{3}}{2}\right)\left(p I_{r e}-C_{m} I_{m p}\right), \\
& \operatorname{EJTP}_{4}\left(Q_{4}, L_{r}, \pi_{x}\right) \\
& =D\left(p-C_{s}-W_{s}-C_{r s} Z_{s}-W_{m}-C_{r m} Z_{m}\right) \\
& -\frac{1}{2} D h_{r} L_{r}\left(\frac{1}{n r}-\frac{\left(\sigma \sqrt{L_{r}} \varphi(k) n_{r} q\right)}{Q_{4}}\right) \\
& -\frac{D}{Q_{4}}\left\{A_{s}+A_{m}+F_{m}+A_{r}+F_{r}\right. \\
& +\left[\frac{\beta_{0}}{\pi_{0}} \pi_{x}^{2}+\pi_{0}-\beta_{0} \pi_{x}\right] \\
& \left.\times \sigma \sqrt{L_{r}} \varphi(k)+C(L)\right\} \\
& -D h_{s}\left[\left(\frac{1}{2}-Z_{s}^{2}\right) \frac{Q_{4}}{P}\right] \\
& -D h_{m}\left(\left(\frac{Q_{4}}{2 D_{r}}\right)-\frac{Z_{m}^{2} Q_{4}}{D_{r}}-\frac{L_{m} n_{m}}{2}\right) \\
& +\frac{\left(Q_{4} X\right)^{2}}{2 Q_{4}}\left(C_{r} I_{m e}-C_{r} I_{s p}\right)
\end{aligned}
$$

$$
\begin{aligned}
& +\frac{\left(Q_{4}-Q_{4} X\right)^{2}}{2 Q_{4}}\left(C_{m} I_{s e}-C_{m} I_{m p}\right) \\
& +\frac{\left(Q_{4} Y\right)^{2}}{2 Q_{4}}\left(p I_{r e}-C_{m} I_{m p}\right) \\
& +\frac{\left(Q_{4}-Q_{4} Y\right)^{2}}{2 Q_{4}}\left(C_{r} I_{m e}-C_{r} I_{r p}\right) .
\end{aligned}
$$

\section{Solution Procedure}

4.1. Determination of the Optimal Backorder Discount $\pi_{x}$ for Any Given $L_{r}$ and $Q_{i}$. We would like to find the minimum value of the joint expected total profit $\operatorname{EJTP}\left(Q_{i}, L_{r}, \pi_{x}\right)$. For any given $Q_{i}$ and $L_{r}$, to take first and second partial derivation of $\operatorname{EJTP}\left(Q_{i}, L_{r}, \pi_{x}\right)$, with respect to $\pi_{x}$, we have

$$
\begin{gathered}
\frac{\partial \operatorname{EJTP}_{i}\left(Q_{i}, L_{r}, \pi_{x}\right)}{\partial \pi_{x}}=\frac{D \sigma \sqrt{L_{r}} \varphi(k)\left(\beta_{0}-2 \beta_{0} \pi_{x} / \pi_{0}\right)}{Q_{i}}, \\
\frac{\partial^{2} \operatorname{EJTP}_{i}\left(Q_{i}, L_{r}, \pi_{x}\right)}{\partial \pi_{x}{ }^{2}}=\frac{-\left(2 \beta_{0} D \sigma \sqrt{L_{r}} \varphi(k)\right)}{Q_{i} \pi_{0}}<0 .
\end{gathered}
$$

Because $(7)<0$, therefore, for fixed $Q_{i}$ and $L_{r}$, $\operatorname{EJTP}\left(Q_{i}, L_{r}, \pi_{x}\right)$ is a concave function in $\pi_{x}$. So as there exists a unique value of $\pi_{x}$ which maximize $\operatorname{EJTP}\left(Q_{i}, L_{r}, \pi_{x}\right), \pi_{x}$ can be obtained by solving the equation $\partial \operatorname{EJTP}\left(Q_{i}, L_{r}, \pi_{x}\right) / \partial \pi_{x}=$ 0 in (6) and is given by

$$
\pi_{x}=\frac{\beta_{0} \pi_{0}-Q_{i} \pi_{0} / D \sigma \sqrt{L_{r}} \varphi(k)}{2 \beta_{0}} .
$$

4.2. Determination of the Optimal Order Quantity $Q_{i}$ for Any Given $L_{r}$ and $\pi_{x}$. For any given $L_{r}$ and $\pi_{x}$, to take first and second partial derivation of $\operatorname{EJTP}\left(Q_{i}, L_{r}, \pi_{x}\right)$, with respect to $Q_{i}$, we have

$$
\begin{aligned}
& \frac{\partial \mathrm{EJTP}_{1}\left(Q_{1}, L_{r}, \pi_{x}\right)}{\partial Q_{1}} \\
& =\left(C_{r} I_{m e}-C_{s} I_{s p}\right)\left(X-\frac{1}{2}\right) \\
& +\left(p I_{r e}-C_{m} I_{m p}\right)\left(Y-\frac{1}{2}\right) \\
& -D h_{m}\left(\frac{1}{2 D_{r}}-\frac{Z_{m}^{2}}{D_{r}}\right)+\frac{D h_{s}\left(Z_{s}^{2}-1 / 2\right)}{P} \\
& +D\left(A_{s}+A_{m}+F_{m}+A_{r}+F_{r}\right. \\
& +\left[\frac{\beta_{0}}{\pi_{0}} \pi_{x}^{2}+\pi_{0}-\beta_{0} \pi_{x}\right] \\
& \left.\times \sigma \sqrt{L_{r}} \varphi(k)+C(L)\right) \times\left(Q_{1}^{2}\right)^{-1} \\
& +\frac{D L_{r}^{3 / 2} h_{r} n_{r} q \sigma \varphi(k)}{2 Q_{1}^{2}},
\end{aligned}
$$


8

Mathematical Problems in Engineering

$$
\begin{aligned}
& \frac{\partial \mathrm{EJTP}_{2}\left(Q_{2}, L_{r}, \pi_{x}\right)}{\partial Q_{2}} \\
& =\left(C_{m} I_{m e}-C_{s} I_{s p}\right)\left(X-\frac{1}{2}\right) \\
& +\frac{1}{2} Y^{2}\left(C_{r} I_{m e}+p I_{r e}-C_{m} I_{m p}-C_{r} I_{r p}\right) \\
& -D h_{m}\left(\frac{1}{2 D_{r}}-\frac{Z_{m}^{2}}{D_{r}}\right)+\frac{D h_{s}\left(Z_{s}^{2}-1 / 2\right)}{P} \\
& +D\left(A_{s}+A_{m}+F_{m}+A_{r}+F_{r}\right. \\
& \quad+\left[\frac{\beta_{0}}{\pi_{0}} \pi_{x}^{2}+\pi_{0}-\beta_{0} \pi_{x}\right] \\
& \left.\quad \times \sigma \sqrt{L_{r}} \varphi(k)+C(L)\right) \times\left(Q_{2}^{2}\right)^{-1} \\
& +\frac{D L_{r}^{3 / 2} h_{r} n_{r} q \sigma \varphi(k)}{2 Q_{2}^{2}},
\end{aligned}
$$

$$
\begin{aligned}
\frac{\partial \mathrm{EJP}_{3}\left(Q_{3}, L_{r}, \pi_{x}\right)}{\partial Q_{3}} & \frac{1}{2} X^{2}\left(C_{r} I_{m e}-C_{s} I_{s p}\right) \\
& +\left(p I_{r e}-C_{m} I_{m p}\right)\left(Y-\frac{1}{2}\right) \\
& -D h_{m}\left(\frac{1}{2 D_{r}}-\frac{Z_{m}^{2}}{D_{r}}\right)+\frac{D h_{s}\left(Z_{s}^{2}-1 / 2\right)}{P} \\
& +D\left(A_{s}+A_{m}+F_{m}+A_{r}+F_{r}\right. \\
& +\left[\frac{\beta_{0}}{\pi_{0}} \pi_{x}^{2}+\pi_{0}-\beta_{0} \pi_{x}\right] \\
& \left.\times \sigma \sqrt{L_{r}} \varphi(k)+C(L)\right) \times\left(Q_{3}^{2}\right)^{-1} \\
& +\frac{D L_{r}^{3 / 2} h_{r} n_{r} q \sigma \varphi(k)}{2 Q_{3}^{2}} \\
& +\frac{\left(C_{m} I_{m p}-C_{m} I_{s e}\right)\left(Q_{3}-Q_{3} X\right)^{2}}{2 Q_{3}^{2}} \\
& +\frac{\left(C_{m} I_{m p}-C_{m} I_{s e}\right)(X-1)\left(Q_{3}-Q_{3} X\right)^{2}}{Q_{3}} \\
\frac{\partial \mathrm{E} J \mathrm{TP}}{4}\left(Q_{4}, L_{r}, \pi_{x}\right) & \frac{1}{2} X^{2}\left(C_{r} I_{m e}-C_{r} I_{s p}\right)
\end{aligned}
$$

$+\frac{1}{2} Y^{2}\left(p I_{r e}-C_{m} I_{m p}\right)$

$-D h_{m}\left(\frac{1}{2 D_{r}}-\frac{Z_{m}^{2}}{D_{r}}\right)+\frac{D h_{s}\left(Z_{s}^{2}-1 / 2\right)}{P}$

$+D\left(A_{s}+A_{m}+F_{m}+A_{r}+F_{r}\right.$

$$
+\left[\frac{\beta_{0}}{\pi_{0}} \pi_{x}^{2}+\pi_{0}-\beta_{0} \pi_{x}\right]
$$$$
\left.\times \sigma \sqrt{L_{r}} \varphi(k)+C(L)\right) \times\left(Q_{4}^{2}\right)^{-1}
$$

$+\frac{D L_{r}^{3 / 2} h_{r} n_{r} q \sigma \varphi(k)}{2 Q_{4}^{2}}$

$+\frac{\left(C_{m} I_{m p}-C_{m} I_{s e}\right)(X-1)\left(Q_{4}-Q_{4} X\right)^{2}}{Q_{4}}$

$-\frac{\left(C_{r} I_{m e}-C_{r} I_{r p}\right)(Y-1)\left(Q_{4}-Q_{4} Y\right)^{2}}{Q_{4}}$

$+\frac{\left(C_{m} I_{m p}-C_{m} I_{s e}\right)\left(Q_{4}-Q_{4} X\right)^{2}}{2 Q_{4}^{2}}$

$-\frac{\left(C_{r} I_{m e}-C_{r} I_{r p}\right)\left(Q_{4}-Q_{4} Y\right)^{2}}{2 Q_{4}^{2}}$,

(9)

$$
\begin{gathered}
\frac{\partial^{2} \operatorname{EJP}_{1}\left(Q_{1}, L_{r}, \pi_{x}\right)}{\partial Q_{1}^{2}} \\
=-2 D\left(A_{s}+A_{m}+F_{m}+A_{r}+F_{r}\right. \\
+\left[\frac{\beta_{0}}{\pi_{0}} \pi_{x}^{2}+\pi_{0}-\beta_{0} \pi_{x}\right] \\
\left.\times \sigma \sqrt{L_{r}} \varphi(k)+C(L)\right) \times\left(Q_{1}^{3}\right)^{-1} \\
-\frac{D L_{r}^{3 / 2} h_{r} n_{r} q \sigma \varphi(k)}{Q_{1}^{3}}<0, \\
\frac{\partial^{2} \mathrm{EJTP}_{2}\left(Q_{2}, L_{r}, \pi_{x}\right)}{\partial Q_{2}^{2}} \\
=-2 D\left(A_{s}+A_{m}+F_{m}+A_{r}+F_{r}\right. \\
+\left[\frac{\beta_{0}}{\pi_{0}} \pi_{x}^{2}+\pi_{0}-\beta_{0} \pi_{x}\right] \\
\left.\times \sigma \sqrt{L_{r}} \varphi(k)+C(L)\right) \times\left(Q_{2}^{3}\right)^{-1} \\
-\frac{D L_{r}^{3 / 2} h_{r} n_{r} q \sigma \varphi(k)}{Q_{2}^{3}}<0,
\end{gathered}
$$




$$
\begin{gathered}
\frac{\partial^{2} \operatorname{EJP}_{3}\left(Q_{3}, L_{r}, \pi_{x}\right)}{\partial Q_{3}^{2}} \\
=-2 D\left(A_{s}+A_{m}+F_{m}+A_{r}+F_{r}\right. \\
\quad+\left[\frac{\beta_{0}}{\pi_{0}} \pi_{x}^{2}+\pi_{0}-\beta_{0} \pi_{x}\right] \\
\left.\times \sigma \sqrt{L_{r}} \varphi(k)+C(L)\right) \times\left(Q_{3}^{3}\right)^{-1} \\
-\frac{D L_{r}^{3 / 2} h_{r} n_{r} q \sigma \varphi(k)}{Q_{3}^{3}}-\frac{\left(C_{m} I_{m p}-C_{m} I_{s e}\right)(X-1)^{2}}{Q_{3}} \\
-\frac{\left(C_{m} I_{m p}-C_{m} I_{s e}\right)\left(Q_{3}-Q_{3} X\right)^{2}}{Q_{3}^{3}} \\
-\frac{2\left(C_{m} I_{m p}-C_{m} I_{s e}\right)(X-1)\left(Q_{3}-Q_{3} X\right)}{Q_{3}^{2}}<0,
\end{gathered}
$$$$
\frac{\partial^{2} \operatorname{EJTP}_{4}\left(Q_{4}, L_{r}, \pi_{x}\right)}{\partial Q_{4}^{2}}
$$$$
=-2 D\left(A_{s}+A_{m}+F_{m}+A_{r}+F_{r}\right.
$$$$
+\left[\frac{\beta_{0}}{\pi_{0}} \pi_{x}^{2}+\pi_{0}-\beta_{0} \pi_{x}\right]
$$$$
\left.\times \sigma \sqrt{L_{r}} \varphi(k)+C(L)\right) \times\left(Q_{4}^{3}\right)^{-1}
$$$$
-\frac{D L_{r}^{3 / 2} h_{r} n_{r} q \sigma \varphi(k)}{Q_{4}^{3}}
$$$$
-\frac{\left(C_{m} I_{m p}-C_{m} I_{s e}\right)(X-1)^{2}}{Q_{4}}
$$$$
-\frac{\left(C_{m} I_{m p}-C_{m} I_{s e}\right)\left(Q_{4}-Q_{4} X\right)^{2}}{Q_{4}^{3}}
$$$$
-\frac{2\left(C_{m} I_{m p}-C_{m} I_{s e}\right)(X-1)\left(Q_{4}-Q_{4} X\right)}{Q_{4}^{2}}
$$$$
+\frac{\left(C_{r} I_{m e}-C_{r} I_{r p}\right)(Y-1)^{2}}{Q_{4}}
$$$$
+\frac{\left(C_{r} I_{m e}-C_{r} I_{r p}\right)\left(Q_{4}-Q_{4} Y\right)^{2}}{Q_{4}^{3}}
$$$$
+\frac{2\left(C_{r} I_{m e}-C_{r} I_{r p}\right)(Y-1)\left(Q_{4}-Q_{4} Y\right)}{Q_{4}^{2}}<0 .
$$

Because $(10)<0$, therefore, for fixed $\pi_{x}$ and $L_{r}$, $\operatorname{EJTP}\left(Q_{i}, L_{r}, \pi_{x}\right)$ is a concave function in $Q_{i}$. So as there exists a unique value of $Q_{i}$ which maximize $\operatorname{EJTP}\left(Q_{i}, L_{r}, \pi_{x}\right), Q_{i}$ can be obtained by solving the equation $\partial \mathrm{EJTP}_{i}\left(Q_{i}, L_{r}, \pi_{x}\right) / \partial Q_{i}=$ 0 in (9) and are given by

$$
\begin{array}{r}
Q_{1}=\left(\left(D \left(A_{s}+A_{m}+F_{m}+A_{r}+F_{r}\right.\right.\right. \\
+\left[\frac{\beta_{0}}{\pi_{0}} \pi_{x}^{2}+\pi_{0}-\beta_{0} \pi_{x}\right] \\
\left.\quad \times \sigma \sqrt{L_{r}} \varphi(k)+C(L)\right) \\
\left.+\frac{D L_{r}^{3 / 2} h_{r} n_{r} q \sigma \varphi(k)}{2}\right) \\
\times\left(-\left(C_{r} I_{m e}-C_{s} I_{s p}\right)\left(X-\frac{1}{2}\right)\right. \\
\quad-\left(p I_{r e}-C_{m} I_{m p}\right)\left(Y-\frac{1}{2}\right) \\
+D h_{m}\left(\frac{1}{2 D_{r}}-\frac{Z_{m}^{2}}{D_{r}}\right)^{-1} \\
\left.\quad-\frac{D h_{s}\left(Z_{s}^{2}-1 / 2\right)}{P}\right)^{1 / 2},
\end{array}
$$

$Q_{2}=\left(\left(D\left(A_{s}+A_{m}+F_{m}+A_{r}+F_{r}\right.\right.\right.$

$$
+\left[\frac{\beta_{0}}{\pi_{0}} \pi_{x}^{2}+\pi_{0}-\beta_{0} \pi_{x}\right]
$$$$
\left.\times \sigma \sqrt{L_{r}} \varphi(k)+C(L)\right)
$$$$
\left.+\frac{D L_{r}^{3 / 2} h_{r} n_{r} q \sigma \varphi(k)}{2}\right)
$$

$$
\times\left(-\left(C_{m} I_{m e}-C_{s} I_{s p}\right)\left(X-\frac{1}{2}\right)\right.
$$$$
-\frac{1}{2} Y^{2}\left(C_{r} I_{m e}+p I_{r e}-C_{m} I_{m p}-C_{r} I_{r p}\right)
$$$$
+D h_{m}\left(\frac{1}{2 D_{r}}-\frac{Z_{m}^{2}}{D_{r}}\right)
$$$$
\left.\left.-\frac{D h_{s}\left(Z_{s}^{2}-1 / 2\right)}{P}\right)^{-1}\right)^{1 / 2} \text {, }
$$

$$
\begin{array}{r}
Q_{3}=\left(\left(D \left(A_{s}+A_{m}+F_{m}+A_{r}+F_{r}\right.\right.\right. \\
+\left[\frac{\beta_{0}}{\pi_{0}} \pi_{x}^{2}+\pi_{0}-\beta_{0} \pi_{x}\right]
\end{array}
$$




$$
\begin{aligned}
& \left.\times \sigma \sqrt{L_{r}} \varphi(k)+C(L)\right) \\
& \left.+\frac{D L_{r}^{3 / 2} h_{r} n_{r} q \sigma \varphi(k)}{2}\right) \\
& \times\left(-\frac{X^{2}\left(C_{r} I_{m e}-C_{s} I_{s p}\right)}{2}\right. \\
& -\left(p I_{r e}-C_{m} I_{m p}\right)\left(Y-\frac{1}{2}\right) \\
& +D h_{m}\left(\frac{1}{2 D_{r}}-\frac{Z_{m}^{2}}{D_{r}}\right) \\
& -\frac{D h_{s}\left(Z_{s}^{2}-1 / 2\right)}{P} \\
& \left.\left.+\frac{\left(C_{m} I_{m p}-C_{m} I_{s e}\right)\left(X^{2}-2 X+1\right)}{2}\right)^{-1}\right)^{1 / 2}, \\
& Q_{4}=\left(\left(D \left(A_{s}+A_{m}+F_{m}+A_{r}+F_{r}\right.\right.\right. \\
& +\left[\frac{\beta_{0}}{\pi_{0}} \pi_{x}^{2}+\pi_{0}-\beta_{0} \pi_{x}\right] \\
& \left.\times \sigma \sqrt{L_{r}} \varphi(k)+C(L)\right) \\
& \left.+\frac{D L_{r}^{3 / 2} h_{r} n_{r} q \sigma \varphi(k)}{2}\right) \\
& \times\left(-\frac{X^{2}\left(C_{r} I_{m e}-C_{s} I_{s p}\right)}{2}\right. \\
& -\frac{Y^{2}\left(p I_{r e}-C_{m} I_{m p}\right)}{2} \\
& +D h_{m}\left(\frac{1}{2 D_{r}}-\frac{Z_{m}^{2}}{D_{r}}\right) \\
& -\frac{D h_{s}\left(Z_{s}^{2}-1 / 2\right)}{P} \\
& +\frac{\left(C_{m} I_{m p}-C_{m} I_{s e}\right)\left(X^{2}-2 X+1\right)}{2} \\
& \left.\left.-\frac{\left(C_{r} I_{m e}-C_{r} I_{r p}\right)\left(Y^{2}-2 Y+1\right)}{2}\right)^{-1}\right)^{1 / 2} \text {. }
\end{aligned}
$$

Algorithm. Summarizing the above arguments, we establish the following algorithm to obtain optimal values of $\operatorname{EJTP}_{i j}\left(Q_{i}, L_{r j}, \pi_{x}\right)$.
Step 1. Arrange the value of $C_{j}$ such that $C_{0} \leq C_{1} \leq C_{2}$.

Step 2. Determine $\pi_{X}$ by solving $(6)=0$.

Step 3. With the different duration, substitute $L_{r j}$ and $\pi_{X}$ into (11) to find $Q_{1}$.

Step 4. Substitute $Q_{1}$ into (5) to find $\mathrm{EJTP}_{1}$.

Step 5. For $j=0,1,2$, repeat Steps 1 to 4 and compute $\operatorname{EJTP}_{1 j}\left(Q_{1}, L_{r j}, \pi_{x}\right)$.

Step 6. For $i=1,2,3,4$ and $j=0,1,2$, repeat Steps 1 to 5 and obtain $\operatorname{EJTP}_{i j}\left(Q_{i}, L_{r j}, \pi_{x}\right)$.

Step 7. Observe $\operatorname{EJTP}_{i j}\left(Q_{i}, L_{r j}, \pi_{x}\right)$ by varying $X$ and $Y$.

Step 8. Determine optimal EJTP ${ }_{i j}\left(Q_{i}, L_{r j}, \pi_{x}\right)$.

\section{Numerical Example}

To illustrate the preceding model, we consider an inventory system with the following data and lead time data in Table 1: $D$ (average demand per year) $=1000$ unit/year, $P$ (production rate of manufacturer $)=10, D_{r}$ (demand rate of retailer $)=8$, $q$ (probability of shortage) $=0.3, \pi_{0}$ (marginal profit per unit) $=\$ 6$ per unit, $\sigma$ (standard deviation) $=7$ unit, $C_{s}$ (supplier's purchasing cost per unit) $=\$ 10$ per unit, $A_{s}$ (supplier's ordering cost per order) $=\$ 100$ per order, $W_{s}$ (supplier's inspecting cost per unit) $=\$ 0.6$ per unit, $C_{r s}$ (supplier's repair cost per unit) $=\$ 0.2$ per unit, $h_{s}$ (supplier's holding cost per unit per production run) $=\$ 2$ per unit, $I_{s p}$ (supplier's capital opportunity cost per dollar per year $)=\$ 0.04$ per year, $I_{s e}$ (supplier's interest earned per dollar per year) $=\$ 0.03$ per year, $Z_{s}$ (probability of imperfect items from supplier) $=0.1$, $C_{m}$ (manufacturer's purchasing cost per unit) $=\$ 25$ per unit, $A_{m}$ (manufacturer's ordering cost per order) $=\$ 120$ per order, $W_{m}$ (manufacturer's inspecting cost per unit) $=\$ 0.6$ per unit, $C_{r m}$ (manufacturer's repair cost per unit) $=\$ 0.25$ per unit, $Z_{m}$ (probability of imperfect items from manufacturer) $=0.1, F_{m}$ (manufacturer's transportation cost per unit $)=\$ 40$ per order, $h_{m}$ (manufacturer's holding cost per unit per production run) $=\$ 2.5$ per unit, $L_{m}$ (lead time of manufacturer $)=7$ days, $I_{m p}$ (manufacturer's capital opportunity cost per dollar per year) $=\$ 0.04$ per year, $I_{m e}$ (manufacturer's interest earned per dollar per year) $=\$ 0.03$ per year, $C_{r}$ (retailer's purchasing cost per unit) $=\$ 30$ per unit, $p$ (retailer's selling price per unit) $=\$ 50$ per unit, $A_{r}$ (retailer's ordering cost per order) $=\$ 150$ per order, $F_{r}$ (retailer's transportation cost per unit) $=\$ 50$ per order, $h_{r}$ (retailer's holding cost per unit per production run) $=\$ 3$ per unit, $I_{r p}$ (retailer's capital opportunity cost per dollar per year) $=\$ 0.04$ per year, and $I_{r e}($ retailer's interest earned per dollar per year $)=\$ 0.03$ per year.

5.1. The Sensitivity Analysis of Decision Variable. First, we need to know how $L_{r}$ and $\pi_{x}$ influence the $\operatorname{EJTP}_{i}\left(Q_{i}, L_{r}, \pi_{x}\right)$ and individual profit, and, according to function (4), we developed four different models, and we could reach the 
TABLE 1: Lead time data of numerical example.

\begin{tabular}{lccc}
\hline $\begin{array}{l}\text { Lead time } \\
\text { component, } j\end{array}$ & $\begin{array}{c}\text { Normal } \\
\text { duration, } T_{j} \\
\text { (days) }\end{array}$ & $\begin{array}{c}\text { Minimum } \\
\text { duration, } t_{j} \text { (days) }\end{array}$ & $\begin{array}{c}\text { Crashing cost, } \\
C_{j} \text { (\$/day) }\end{array}$ \\
\hline 1 & 20 & 14 & 1.2 \\
2 & 16 & 10 & 5 \\
\hline
\end{tabular}

optimal $\operatorname{EJTP}_{i}\left(Q_{i}, L_{r}, \pi_{x}\right)$ by applying the algorithm; in addition, we take the $\operatorname{EJTP}_{1}\left(Q_{1}, L_{r}, \pi_{x}\right)$ as an example here; the results are shown in Table 2.

The results are shown in Table 2; we could observe how lead time of retailer $L_{r}$ and backorder discount $\pi_{x}$ influenced the $\operatorname{EJTP}_{1}\left(Q_{1}, L_{r}, \pi_{x}\right)$ and individual profit. The total profit of $\operatorname{EJTP}_{1}\left(Q_{1}, L_{r}, \pi_{x}\right)$ decreased with longer lead time of retailer $L_{r}$, and the profit of retailer increased with longer lead time $L_{r}$. We might indicate that performance of $\operatorname{EJTP}_{1}\left(Q_{1}, L_{r}, \pi_{x}\right)$ could be enhanced by compressing the lead time but it also caused lower profit for the retailer. The profit of $\operatorname{EJTP}_{1}\left(Q_{1}, L_{r}, \pi_{x}\right)$ and retailer could reach the optimal results when $\pi_{x}=3$; it is suggested that backorder discount should be offered appropriately to the buyer. Owing to the fact that the expected joint total annual profit was greater than individual's profit (supplier + manufacturer + retailer) and shorter lead time was more benefit for $\operatorname{EJTP}_{1}\left(Q_{1}, L_{r}, \pi_{x}\right)$. It seems that compressing the lead time was an available policy in our proposed inventory models. And we continue discussing the more detailed inventory policy in the following sections.

5.2. The Permissible Delay Period $X$ and EJTP. Let us observe the value of profit with $\beta_{0}=0.8$ by varying the permissible delay period $X$, and, with applying the proposed procedure, the solution results are contained in Figure 4 (see Appendix B).

To compare which condition is more beneficial to our proposed inventory model, we have more detailed results in Table 3.

According to Section 3.1, we knew that, if payment time was longer than lead time, then it would bring additional interest income to the buyer. On the other hand, if payment time was shorter than lead time, it would cause buyer to bear additional opportunity cost. From the above discussion, we had four possible cases in $\operatorname{EJTP}_{i}\left(Q_{i}, L_{r}, \pi_{x}\right)$, for $i=1,2,3,4$, and considered the different duration of $L_{r j}$, for $j=0,1,2$. Hence, we had twelve possible values of profit. According to the data from Table 3, we could indicate that the optimal profit occurred in $\operatorname{EJTP}_{4}\left(Q_{4}, L_{r}, \pi_{x}\right)$ with normal duration condition, and the worst profit occurred in $\mathrm{EJTP}_{3}$ with normal duration condition.

The Hessian matrix $\mathrm{H}$ at the optimal solution $Q_{4}^{*}=92.06$, $L_{r}^{*}=36$, and $\pi_{x}^{*}=2.96489$ is $\left[\begin{array}{lll}-3.34166 & -3.83811 & -0.01862 \\ -3.83811 & -4.90745 & 0.0138882 \\ -0.01862 & 0.0138882 & -28.4806\end{array}\right]$ which is negative definite because all the eigenvalues are negative. Hence, the required optimal solution is $Q_{4}^{*}=92.06$, $L_{r}^{*}=36$, and $\pi_{x}^{*}=2.96489$ and $\operatorname{EJTP}_{4}^{*}\left(Q_{4}, L_{r}, \pi_{x}\right)=20678.1$.
5.3. The Permissible Delay Period $Y$ and EJTP. In this section, we observe the value of profit by varying the permissible delay period $Y$. The detailed results have been shown in Table 4 .

This time, we varied the permissible delay period $Y$. We noticed that the optimal profit still occurred in $\operatorname{EJTP}_{4}\left(Q_{4}, L_{r}, \pi_{x}\right)$ with normal duration condition. But the worst profit was different from previous result; it occurred in $\operatorname{EJTP}_{4}\left(Q_{4}, L_{r}, \pi_{x}\right)$ with minimum duration condition.

The Hessian matrix $\mathrm{H}$ at the optimal solution $Q_{4}^{*}=87.55$, $L_{r}^{*}=36$, and $\pi_{x}^{*}=2.96661$ is $\left[\begin{array}{lll}-3.88515 & -4.24373 & -0.01142 \\ -4.24373 & -5.16025 & 0.0138882 \\ -0.01142 & 0.0138882 & -29.9477\end{array}\right]$ which is negative definite because all the eigenvalues are negative. Hence, the required optimal solution is $Q_{4}^{*}=87.55$, $L_{r}^{*}=36$, and $\pi_{x}^{*}=2.96661$ and $\operatorname{EJTP}_{4}^{*}\left(Q_{4}, L_{r}, \pi_{x}\right)=20313.7$.

No matter which payment time we varied, the optimal profit still occurred in the same condition. Hence, through controllable lead time and payment time, we could find the optimal policy in our proposed integrated inventory model.

\section{Conclusions and Future Works}

In this paper, we discussed the issue of a three-echelon inventory model with permissible delay in payments under controllable lead time and backorder consideration to find out the suitable inventory policy to enhance profit of the supply chain. Owing to information revolution, the community can get anything they want easily. It causes that any goods in the market always have a stable price due to the inspection by community. Hence, the enterprises are hardly raising the price on their product. If they want to enhance the profit, to control the relevant cost and reduce it efficiently is a good approach to enhance profit for them.

In the manufacture part, the firms could delay the payment time to obtain additional interest income, and the proper payment time could be a great benefit for the firms. In the customer part, length of lead time usually influenced the price of products. Fast lead time could respond to customer's demand quickly, and firms could request customers for high price. Inevitably, firms must pay the additional cost for reducing lead time. In the stock part, firms usually adopted backorder approach to minimize sales loss and offered some proper discount to save customers.

From the above discussion, we proposed our inventory model and analyzed its profits, in a three-echelon inventory model case. First, we observed that integrated profit is greater than individual's profit. Also we concluded that the compressed lead time and appropriate backorder discount are most benefit factors for entire supply chain in Section 5.1. Second, we fixed the backorder ratio and observed the optimal profit by varying payment time in Sections 5.2 and 5.3. With the different duration and four cases in $\operatorname{EJTP}_{i}\left(Q_{i}, L_{r}, \pi_{x}\right)$, we had twelve possible values of profit, and the optimal profit occurred in $\operatorname{EJTP}_{4}\left(Q_{4}, L_{r}, \pi_{x}\right)$ with normal duration. Finally, we discover that the profit of supply chain will be different by compressing lead time and varying payment time.

In the supply chain, with the real data as input parameters, our proposed model can help decision-makers enhance the performance of the supply chain by controlling lead time and payment time. As far as the authors' knowledge goes, no such 
TABLE 2: The profit of $\operatorname{EJTP}_{1}\left(Q_{1}, L_{r}, \pi_{x}\right)$ and individual.

\begin{tabular}{lccccccc}
\hline$D$ & $L_{r}$ & $Q_{1}$ & $\mathrm{EJTP}_{1}\left(Q_{1}, L_{r}, \pi_{x}\right)$ & $D$ & $\pi_{x}$ & $Q_{1}$ & $\mathrm{EJTP}_{1}\left(Q_{1}, L_{r}, \pi_{x}\right)$ \\
\hline${ }^{*} 1 \mathrm{~K}$ & 15 & 55.4568 & ${ }^{*} 16366.8507$ & $1 \mathrm{~K}$ & 1 & 75.5802 & 14194.8814 \\
$1 \mathrm{~K}$ & 20 & 60.0695 & 16068.2148 & $1 \mathrm{~K}$ & 2 & 75.4669 & 14278.6926 \\
$1 \mathrm{~K}$ & 25 & 64.8780 & 15662.1435 & $1 \mathrm{~K}$ & 3 & 75.4287 & ${ }^{*} 14306.6807$ \\
$1 \mathrm{~K}$ & 30 & 69.7979 & 15139.5899 & $1 \mathrm{~K}$ & 4 & 75.4666 & 14278.6926 \\
$1 \mathrm{~K}$ & 35 & 74.7770 & 14507.1236 & $1 \mathrm{~K}$ & 5 & 75.5802 & $Q_{1}$ \\
\hline$D$ & $L_{r}$ & $Q_{1}$ & Supplier & $D$ & $\pi_{x}$ & 14194.8814 \\
\hline $1 \mathrm{~K}$ & $15 \sim 35$ & 10.0861 & 3446.6683 & $1 \mathrm{~K}$ & $1 \sim 5$ & 10.0861 & 3446.6683 \\
\hline$D$ & $L_{r}$ & $Q_{1}$ & Manufacturer & $D$ & $\pi_{x}$ & $Q_{1}$ & Manufacturer \\
\hline $1 \mathrm{~K}$ & $15 \sim 35$ & 6.3162 & -13561.9435 & $1 \mathrm{~K}$ & $1 \sim 5$ & 6.3162 & -13561.9435 \\
\hline$D$ & $L_{r}$ & $Q_{1}$ & Retailer & $D$ & $\pi_{x}$ & $Q_{1}$ & Retailer \\
\hline $1 \mathrm{~K}$ & 15 & 15.0492 & 16870.0034 & $1 \mathrm{~K}$ & 1 & 104.3418 & 20571.7856 \\
$1 \mathrm{~K}$ & 20 & 48.3484 & 19208.4841 & $1 \mathrm{~K}$ & 2 & 104.2893 & 20572.1597 \\
$1 \mathrm{~K}$ & 25 & 68.7905 & 19972.7063 & $1 \mathrm{~K}$ & 3 & 104.2134 & $* 20572.2606$ \\
$1 \mathrm{~K}$ & 30 & 85.9062 & 20359.0393 & $1 \mathrm{~K}$ & 4 & 104.1140 & 20572.0941 \\
$1 \mathrm{~K}$ & 35 & 101.2845 & $* 20550.3897$ & $1 \mathrm{~K}$ & 5 & 103.9911 & 20571.6672 \\
\hline
\end{tabular}

* Optimal solution of EJTP.

${ }^{*} 1 \mathrm{~K}=1000$.

TABLE 3: The value of profit in different conditions by varying $X$.

\begin{tabular}{lccc}
\hline$\beta_{0}=0.8$ & $L_{r 0}$ & $L_{r 1}$ & $L_{r 2}$ \\
\hline$Q_{1}$ & $75.43 \sim 85.19$ & $69.34 \sim 78.18$ & $64.2 \sim 72.26$ \\
$X$ & $8 \sim 107$ & $8 \sim 107$ & $8 \sim 107$ \\
$\operatorname{EJTP}_{1}\left(Q_{1}, L_{r}, \pi_{x}\right)$ & $14306.68 \sim 15847.64$ & $14837.16 \sim 16500.41$ & $14666.7 \sim 16527.05$ \\
\hline$Q_{2}$ & $104.71 \sim 124.4$ & $83.92 \sim 94.93$ & $71.26 \sim 78.76$ \\
$X$ & $8 \sim 107$ & $8 \sim 107$ & $8 \sim 107$ \\
$\operatorname{EJTP}_{2}\left(Q_{2}, L_{r}, \pi_{x}\right)$ & $18067.25 \sim 19600.13$ & $17393.2 \sim 18804.44$ & $16317.84 \sim 17750.81$ \\
\hline$Q_{3}$ & $76.1 \sim 74.8$ & $69.88 \sim 68.7$ & $63.62 \sim 64.69$ \\
$X$ & $7 \sim 0$ & $7 \sim 0$ & $7 \sim 0$ \\
$\operatorname{EJTP}_{3}\left(Q_{3}, L_{r}, \pi_{x}\right)$ & $14144.93 \sim 14200.08$ & $14691.76 \sim 14711.56$ & $7567512.15 \sim 14553.64$ \\
\hline$Q_{4}$ & $87.56 \sim 92.06$ & $75.83 \sim 78.7$ & $7 \sim 0$ \\
$X$ & $7 \sim 0$ & $7 \sim 91 \sim 72.07$ & $7 \sim 0$ \\
$\operatorname{EJTP}_{4}\left(Q_{4}, L_{r}, \pi_{x}\right)$ & $* 20313.71 \sim 20678.1$ & $18369.36 \sim 18776.31$ & $16028.2 \sim 16488.16$ \\
\hline
\end{tabular}

* Optimal solution of $\mathrm{EJTP}_{i j}$.

TABLE 4: The value of profit in different conditions by varying $Y$.

\begin{tabular}{lccc}
\hline$\beta_{0}=0.8$ & $L_{r 0}$ & $L_{r 1}$ & $L_{r 2}$ \\
\hline$Q_{1}$ & $75.43 \sim 85.19$ & $69.34 \sim 78.18$ & $64.21 \sim 72.26$ \\
$Y$ & $37 \sim 136$ & $31 \sim 130$ & $25 \sim 124$ \\
$\operatorname{EJTP}_{1}\left(Q_{1}, L_{r}, \pi_{x}\right)$ & $14306.68 \sim 15847.64$ & $14837.2 \sim 16500.4$ & $14666.7 \sim 16527.05$ \\
\hline$Q_{2}$ & $72.43 \sim 104.71$ & $67.02 \sim 83.92$ & $62.45 \sim 71.26$ \\
$Y$ & $36 \sim 0$ & $30 \sim 0$ & $24 \sim 0$ \\
$\operatorname{EJTP}_{2}\left(Q_{2}, L_{r}, \pi_{x}\right)$ & $13749.61 \sim 18067.25$ & $14326.88 \sim 17393.2$ & $14198.61 \sim 16317.84$ \\
\hline$Q_{3}$ & $76.1 \sim 86.16$ & $69.87 \sim 78.94$ & $64.69 \sim 72.95$ \\
$Y$ & $37 \sim 136$ & $30 \sim 129$ & $24 \sim 123$ \\
$\operatorname{EJTP}_{3}\left(Q_{3}, L_{r}, \pi_{x}\right)$ & $14144.93 \sim 15664.35$ & $14691.76 \sim 16336.02$ & $14553.64 \sim 16399.41$ \\
\hline$Q_{4}$ & $59.79 \sim 87.55$ & $59.89 \sim 75.83$ & $60.69 \sim 69.91$ \\
$Y$ & $36 \sim 0$ & $30 \sim 0$ & $24 \sim 0$ \\
$\operatorname{EJTP}_{4}\left(Q_{4}, L_{r}, \pi_{x}\right)$ & $* 16859.1 \sim 20313.7$ & $15392.02 \sim 18369.36$ & $13701.0 \sim 16028.2$ \\
\hline
\end{tabular}

${ }^{*}$ Optimal solution of $\mathrm{EJTP}_{i j}$. 

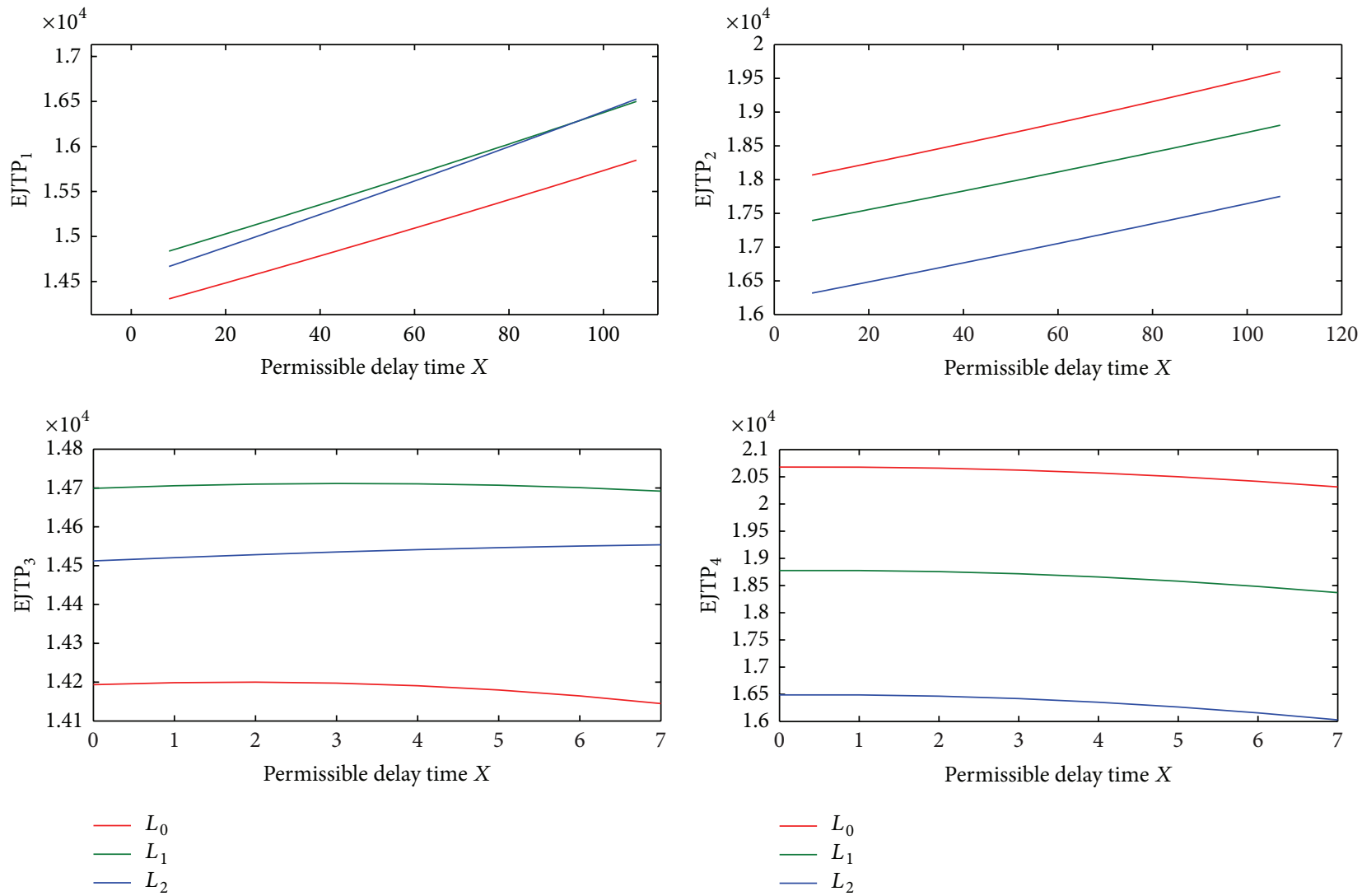

FIgURE 4: The value of profit by change $X$ in $\mathrm{EJTP}_{i}$, for $i=1,2,3,4$.

type of three-echelon inventory model involving controllable lead time has yet been discussed in relevant literatures. These are the new major contributions of the present paper.

Finally, it is our hope that this work will encourage future work in this area and related areas. And we will improve our further research in more real-world complexities, such as the concept of present value, multiple vendor and buyer, and apply the fuzzy approach into the proposed models, and we will cooperate real case to get the actual data that can illustrate real numerical examples to our future research.

\section{Appendices}

\section{A. Inventory Level}

See Figures 1, 2, and 3.

\section{B. Profit Variation}

See Figure 4.

\section{Proof}

The following test can be applied at a nondegenerate critical point $x$. If the Hessian is positive definite at $x$, then $f$ attains a local minimum at $x$. If the Hessian is negative definite at $x$, then $f$ attains a local maximum at $x$. If the
Hessian has both positive and negative eigenvalues, then $x$ is a saddle point for $f$ (this is true even if $x$ is degenerate). Otherwise the test is inconclusive. If all the principal minor of $\mathrm{H}\left(\left|\mathrm{H}_{11}\right|,\left|\mathrm{H}_{22}\right|\right.$ and $\left.\left|\mathrm{H}_{33}\right|\right)>0$, the Hessian matrix $\mathrm{H}$ is positive definite at $\left(Q_{i}, L_{r}, \pi_{x}\right)$; it also means there will be a local minimum at $\left(Q_{i}, L_{r}, \pi_{x}\right)$; if all the principal minor of $\mathrm{H}\left(\left|\mathrm{H}_{11}\right|,\left|\mathrm{H}_{22}\right|\right.$ and $\left.\left|\mathrm{H}_{33}\right|\right)<0$, the Hessian matrix $\mathrm{H}$ is negative definite at $\left(Q_{i}, L_{r}, \pi_{x}\right)$; it also means there will be a local maximum at $\left(Q_{i}, L_{r}, \pi_{x}\right)$.

The Hessian matrix $\mathrm{H}$ of $\operatorname{EJTP}_{i}\left(Q_{i}, L_{r}, \pi_{x}\right)$ can be shown as

$\mathrm{H}$

$$
=\left[\begin{array}{lll}
\frac{\partial^{2} \mathrm{EJTP}_{i}\left(Q_{i}, L_{r}, \pi_{x}\right)}{\partial Q_{i}^{2}} & \frac{\partial^{2} \operatorname{EJTP}_{i}\left(Q_{i}, L_{r}, \pi_{x}\right)}{\partial \mathrm{Q} \partial L_{r}} & \frac{\partial^{2} \operatorname{EJTP}_{i}\left(Q_{i}, L_{r}, \pi_{x}\right)}{\partial \mathrm{Q} \partial \pi_{x}} \\
\frac{\partial^{2} \operatorname{EJTP}_{i}\left(Q_{i}, L_{r}, \pi_{x}\right)}{\partial L_{r} \partial Q} & \frac{\partial^{2} \operatorname{EJTP}_{i}\left(Q_{i}, L_{r}, \pi_{x}\right)}{\partial L_{r}^{2}} & \frac{\partial^{2} \operatorname{EJTP}_{i}\left(Q_{i}, L_{r}, \pi_{x}\right)}{\partial \pi_{x} \partial L_{r}} \\
\frac{\partial^{2} \operatorname{EJTP}_{i}\left(Q_{i}, L_{r}, \pi_{x}\right)}{\partial \pi_{x} \partial Q} & \frac{\partial^{2} \operatorname{EJTP}_{i}\left(Q_{i}, L_{r}, \pi_{x}\right)}{\partial L_{r} \partial \pi_{x}} & \frac{\partial^{2} \operatorname{EJTP}_{i}\left(Q_{i}, L_{r}, \pi_{x}\right)}{\partial \pi_{x}^{2}}
\end{array}\right],
$$

where

$$
\begin{aligned}
& \frac{\partial^{2} \operatorname{EJTP}_{i}\left(Q_{i}, L_{r}, \pi_{x}\right)}{\partial Q_{i}^{2}} \\
& \quad=\left(-2 D\left(A_{s}+A_{m}+F_{m}+A_{r}+F_{r}\right.\right.
\end{aligned}
$$




$$
\begin{aligned}
& +\left[\frac{\beta_{0}}{\pi_{0}} \pi_{x}^{2}+\pi_{0}-\beta_{0} \pi_{x}\right] \\
& \left.\left.\times \sigma \sqrt{L_{r}} \varphi(k)+C(L)\right)\right) \times\left(Q_{i}^{3}\right)^{-1} \\
& -\frac{D L_{r}^{3 / 2} h_{r} n_{r} q \sigma \varphi(k)}{Q_{i}^{3}}
\end{aligned}
$$

$$
\begin{aligned}
& \frac{\partial^{2} \operatorname{EJTP}_{i}\left(Q_{i}, L_{r}, \pi_{x}\right)}{\partial L_{r}^{2}} \\
& =\frac{D \sigma \varphi(k)\left[\left(\beta_{0} / \pi_{0}\right) \pi_{x}^{2}+\pi_{0}-\beta_{0} \pi_{x}\right]}{4 Q L_{r}^{3 / 2}}-\frac{3 D h_{r} n_{r} q \sigma \varphi(k)}{8 Q \sqrt{L_{r}}}, \\
& \frac{\partial^{2} \operatorname{EJTP}_{i}\left(Q_{i}, L_{r}, \pi_{x}\right)}{\partial \pi_{x}^{2}}=\frac{-\left(2 \beta_{0} D \sigma \sqrt{L_{r}} \varphi(k)\right)}{Q_{i} \pi_{0}}, \\
& \frac{\partial^{2} \operatorname{EJTP}_{i}\left(Q_{i}, L_{r}, \pi_{x}\right)}{\partial Q \partial L_{r}}=\frac{\partial^{2} \operatorname{EJTP}_{i}\left(Q_{i}, L_{r}, \pi_{x}\right)}{\partial L_{r} \partial Q} \\
& =\frac{D \sigma \varphi(k)\left[\left(\beta_{0} / \pi_{0}\right) \pi_{x}^{2}+\pi_{0}-\beta_{0} \pi_{x}\right]}{2 Q^{2} \sqrt{L_{r}}} \\
& -\frac{3 D \sqrt{L_{r}} h_{r} n_{r} q \sigma \varphi(k)}{4 Q^{2}} \\
& \frac{\partial^{2} \operatorname{EJTP}_{i}\left(Q_{i}, L_{r}, \pi_{x}\right)}{\partial Q \partial \pi_{x}}=\frac{\partial^{2} \operatorname{EJTP}_{i}\left(Q_{i}, L_{r}, \pi_{x}\right)}{\partial \pi_{x} \partial Q} \\
& =\frac{-\left(D \sqrt{L_{r}} \sigma \varphi(k)\left(\beta_{0}-2 \beta_{0} \pi_{x} / \pi_{0}\right)\right)}{Q^{2}}, \\
& \frac{\partial^{2} \operatorname{EJTP}_{i}\left(Q_{i}, L_{r}, \pi_{x}\right)}{\partial L_{r} \partial \pi_{x}}=\frac{\partial^{2} \operatorname{EJTP}_{i}\left(Q_{i}, L_{r}, \pi_{x}\right)}{\partial \pi_{x} \partial L_{r}} \\
& =\frac{D \sigma \varphi(k)\left(\beta_{0}-2 \beta_{0} \pi_{x} / \pi_{0}\right)}{2 Q \sqrt{L_{r}}} .
\end{aligned}
$$

Next, we can evaluate the principal minor of $\mathrm{H}$ at point $\left(Q_{i}, L_{r}, \pi_{x}\right)$; the first principal minor of $\mathrm{H}$ is

$$
\begin{aligned}
& \left|\mathrm{H}_{11}\right| \\
& =\left(-2 D\left(A_{s}+A_{m}+F_{m}+A_{r}+F_{r}\right.\right. \\
& +\left[\frac{\beta_{0}}{\pi_{0}} \pi_{x}^{2}+\pi_{0}-\beta_{0} \pi_{x}\right] \\
& \left.\left.\quad \times \sigma \sqrt{L_{r}} \varphi(k)+C(L)\right)\right) \times\left(Q_{i}^{3}\right)^{-1} \\
& -\frac{D L_{r}^{3 / 2} h_{r} n_{r} q \sigma \varphi(k)}{Q_{i}^{3}}<0 .
\end{aligned}
$$

The second principal minor of $\mathrm{H}$ is

$$
\begin{gathered}
\left|\mathrm{H}_{22}\right| \\
=\left[\frac{D \sigma \varphi(k)\left(\left(\beta_{0} / \pi_{0}\right) \pi_{x}^{2}+\pi_{0}-\beta_{0} \pi_{x}\right)}{4 Q_{i} L_{r}^{3 / 2}}\right. \\
\left.\quad-\frac{3 D h_{r} n_{r} q \sigma \varphi(k)}{8 Q_{i} \sqrt{L_{r}}}\right] \\
\times-\left[\left(2 D \left(A_{s}+A_{m}+F_{m}+A_{r}+F_{r}\right.\right.\right. \\
+\left[\frac{\beta_{0}}{\pi_{0}} \pi_{x}^{2}+\pi_{0}-\beta_{0} \pi_{x}\right] \\
\left.\left.\quad \times \sigma \sqrt{L_{r}} \varphi(k)+C(L)\right)\right) \times\left(Q_{i}^{3}\right)^{-1} \\
\left.+\frac{D L_{r}^{3 / 2} h_{r} n_{r} q \sigma \varphi(k)}{Q_{i}^{3}}\right]^{3} \\
-\left[\frac{D \sigma \varphi(k)\left(\left(\beta_{0} / \pi_{0}\right) \pi_{x}^{2}+\pi_{0}-\beta_{0} \pi_{x}\right)}{2 Q_{i}^{2} \sqrt{L_{r}}}\right. \\
\left.\quad-\frac{3 D \sqrt{L_{r}} h_{r} n_{r} q \sigma \varphi(k)}{4 Q_{i}^{2}}\right]^{2}<0 .
\end{gathered}
$$

And the third principal minor of $\mathrm{H}$ is

$$
\begin{gathered}
\left|\mathrm{H}_{33}\right| \\
=\frac{1}{\mathrm{Q}^{4}}\left\{-\frac{1}{4} \frac{1}{L_{r}} \mathrm{Q}^{2} D^{2} \sigma^{2} \varphi(k)^{2}\left(\beta_{0}-\frac{2 \beta_{0} \pi_{x}}{\pi_{0}}\right)^{2}\right. \\
\times\left(2 D \left(A_{s}+A_{m}+F_{m}+A_{r}+F_{r}\right.\right. \\
+\left[\frac{\beta_{0}}{\pi_{0}} \pi_{x}^{2}+\pi_{0}-\beta_{0} \pi_{x}\right] \\
\left.\times \sigma \sqrt{L_{r}} \varphi(k)+C(L)\right) \times\left(Q_{i}^{3}\right)^{-1} \\
\left.+\frac{D L_{r}^{3 / 2} h_{r} n_{r} q \sigma \varphi(k)}{Q_{i}^{3}}\right) \\
-Q^{2} D^{2} \sigma^{2} \varphi(k)^{2}\left(\beta_{0}-\frac{2 \beta_{0} \pi_{x}}{\pi_{0}}\right)^{2} \\
\times\left(\frac{D\left[\left(\beta_{0} / \pi_{0}\right) \pi_{x}^{2}+\pi_{0}-\beta_{0} \pi_{x}\right] \sigma \sqrt{L_{r}} \varphi(k)}{2 Q_{i}^{2} \sqrt{L_{r}}}\right. \\
\left.+\frac{3 D \sqrt{L_{r}} h_{r} n_{r} q \sigma \varphi(k)}{4 Q_{i}^{2}}\right)
\end{gathered}
$$




$$
\begin{aligned}
& -L_{r} D^{2} \sigma^{2} \varphi(k)^{2}\left(\beta_{0}-\frac{2 \beta_{0} \pi_{x}}{\pi_{0}}\right)^{2} \\
& \times\left(\frac{D\left[\left(\beta_{0} / \pi_{0}\right) \pi_{x}^{2}+\pi_{0}-\beta_{0} \pi_{x}\right] \sigma \sqrt{L_{r}} \varphi(k)}{4 Q_{i} L_{r}^{3 / 2}}\right. \\
& \left.+\frac{3 D h_{r} n_{r} q \sigma \varphi(k)}{8 Q_{i} \sqrt{L_{r}}}\right) \\
& -\frac{1}{\pi_{0}} Q^{3} 2 \beta_{0} D \sigma \sqrt{L_{r}} \varphi(k) \\
& \times\left[\left(2 D \left(A_{s}+A_{m}+F_{m}+A_{r}+F_{r}\right.\right.\right. \\
& +\left[\frac{\beta_{0}}{\pi_{0}} \pi_{x}^{2}+\pi_{0}-\beta_{0} \pi_{x}\right] \\
& \left.\times \sigma \sqrt{L_{r}} \varphi(k)+C(L)\right) \times\left(Q_{i}^{3}\right)^{-1} \\
& \left.+\frac{D L_{r}^{3 / 2} h_{r} n_{r} q \sigma \varphi(k)}{Q_{i}^{3}}\right) \\
& \times\left(\frac{D\left[\left(\beta_{0} / \pi_{0}\right) \pi_{x}^{2}+\pi_{0}-\beta_{0} \pi_{x}\right] \sigma \sqrt{L_{r}} \varphi(k)}{4 Q_{i} L_{r}^{3 / 2}}\right. \\
& \left.+\frac{3 D h_{r} n_{r} q \sigma \varphi(k)}{8 Q_{i} \sqrt{L_{r}}}\right) \\
& +\left(\frac{D\left[\left(\beta_{0} / \pi_{0}\right) \pi_{x}^{2}+\pi_{0}-\beta_{0} \pi_{x}\right] \sigma \sqrt{L_{r}} \varphi(k)}{2 Q_{i}^{2} \sqrt{L_{r}}}\right. \\
& \left.\left.\left.+\frac{3 D \sqrt{L_{r}} h_{r} n_{r} q \sigma \varphi(k)}{4 Q_{i}^{2}}\right)\right]\right\} \text {, }
\end{aligned}
$$

where

$$
\frac{\beta_{0}}{\pi_{0}} \pi_{x}^{2}+\pi_{0}-\beta_{0} \pi_{x}=\pi_{0}\left(4-\beta_{0}\right) .
$$

With $0<\beta_{0}<1$, we have (C.6) $>0$ and, substituting it into (C.5), we can obtain $\left|\mathrm{H}_{33}\right|<0$. Therefore, from (C.3) to (C.5), it follows that the Hessian matrix $\mathrm{H}$ is negative definite at $\left(Q_{i}, L_{r}, \pi_{x}\right)$.

\section{Conflict of Interests}

None of the authors has any conflict of interests regarding this study.

\section{Acknowledgments}

The authors would like to thank the editors and referees for their helpful comments and suggestions.

\section{References}

[1] H. M. Beheshti, "A decision support system for improving performance of inventory management in a supply chain network," International Journal of Productivity and Performance Management, vol. 59, no. 5, pp. 452-467, 2010.

[2] W. Jammernegg and G. Reiner, "Performance improvement of supply chain processes by coordinated inventory and capacity management," International Journal of Production Economics, vol. 108, no. 1-2, pp. 183-190, 2007.

[3] G. P. Cachon and M. Fisher, "Supply chain inventory management and the value of shared information," Management Science, vol. 46, no. 8, pp. 1032-1048, 2000.

[4] D. L. Olson and M. Xie, "A comparison of coordinated supply chain inventory management systems," International Journal of Services and Operations Management, vol. 6, no. 1, pp. 73-88, 2010.

[5] R. Frankel, "The role and relevance of refocused inventory: supply chain management solutions," Business Horizons, vol. 49, no. 4, pp. 275-286, 2006.

[6] M. Das Roy, S. S. Sana, and K. Chaudhuri, "An optimal shipment strategy for imperfect items in a stock-out situation," Mathematical and Computer Modelling, vol. 54, no. 9-10, pp. 2528-2543, 2011.

[7] B. Maddah and M. Y. Jaber, "Economic order quantity for items with imperfect quality: revisited," International Journal of Production Economics, vol. 112, no. 2, pp. 808-815, 2008.

[8] S. S. Sana, "An EOQ model for stochastic demand for limited capacity of own warehouse," Annals of Operations Research, 2013.

[9] G. R. Nasiri, H. Davoudpour, and B. Karimi, "The impact of integrated analysis on supply chain management: a coordinated approach for inventory control policy," Supply Chain Management, vol. 15, no. 4, pp. 277-289, 2010.

[10] M.-C. Lo, J. C.-H. Pan, K.-C. Lin, and J.-W. Hsu, "Impact of lead time and safety factor in mixed inventory models with backorder discounts," Journal of Applied Sciences, vol. 8, no. 3, pp. 528-533, 2008.

[11] J. C.-H. Pan, M.-C. Lo, and Y.-C. Hsiao, "Optimal reorder point inventory models with variable lead time and backorder discount considerations," European Journal of Operational Research, vol. 158, no. 2, pp. 488-505, 2004.

[12] L.-Y. Ouyang, B.-R. Chuang, and Y.-J. Lin, "Impact of backorder discounts on periodic review inventory model," International Journal of Information and Management Sciences, vol. 14, no. 3, pp. 1-13, 2003.

[13] J. C.-H. Pan and Y.-C. Hsiao, "Integrated inventory models with controllable lead time and backorder discount considerations," International Journal of Production Economics, vol. 93-94, pp. 387-397, 2005.

[14] C. Chandra and J. Grabis, "Inventory management with variable lead-time dependent procurement cost," Omega, vol. 36, no. 5, pp. 877-887, 2008.

[15] M. Ben-Daya and A. Raouf, "Inventory models involving lead time as a decision variable," Journal of the Operational Research Society, vol. 45, no. 5, pp. 579-582, 1994.

[16] H. Y. Xia and P. Q. Huang, "An inventory model with quantity discounts and variable lead time when demand distribution is unknown," Journal of Shanghai Jiaotong University, vol. 42, no. 9, pp. 1515-1519, 2008. 
[17] J.-S. Song, H. Zhang, Y. Hou, and M. Wang, "The effect of lead time and demand uncertainties in $(r, q)$ inventory systems," Operations Research, vol. 58, no. 1, pp. 68-80, 2010.

[18] M.-C. Lo and M.-F. Yang, "Imperfect reworking process consideration in integrated inventory model under permissible delay in payments," Mathematical Problems in Engineering, vol. 2008, Article ID 396252, 18 pages, 2008.

[19] L.-H. Chen and F.-S. Kang, "Integrated vendor-buyer cooperative inventory models with variant permissible delay in payments," European Journal of Operational Research, vol. 183, no. 2, pp. 658-673, 2007.

[20] L. Zhu, J. Zhang, and J. Zhang, "An inventory model with limited storage capacity and shortages under permissible delay in payments," in Proceedings of the 8th International Conference of Chinese Logistics and Transportation Professionals-Logistics: The Emerging Frontiers of Transportation and Development in China, pp. 1489-1495, August 2008.

[21] C.-K. Huang, D.-M. Tsai, J.-C. Wu, and K.-J. Chung, "An optimal integrated vendorbuyer inventory policy under conditions of order-processing time reduction and permissible delay in payments," International Journal of Production Economics, vol. 128, no. 1, pp. 445-451, 2010.

[22] S. S. Sana and K. S. Chaudhuri, "A deterministic EOQ model with delays in payments and price-discount offers," European Journal of Operational Research, vol. 184, no. 2, pp. 509-533, 2008.

[23] A. Roy, S. S. Sana, and K. Chaudhuri, "Optimal replenishment order for uncertain demand in three layer supply chain," Economic Modelling, vol. 29, no. 6, pp. 2274-2282, 2012.

[24] S. S. Sana, "A collaborating inventory model in a supply chain," Economic Modelling, vol. 29, no. 5, pp. 2016-2023, 2012.

[25] B. Pal, S. S. Sana, and K. Chaudhuri, "A three layer multiitem production-inventory model for multiple suppliers and retailers," Economic Modelling, vol. 29, no. 6, pp. 2704-2710, 2012.

[26] S. S. Sana, J. A. Chedid, and K. S. Navarro, "A three layer supply chain model with multiple suppliers, manufacturers and retailers for multiple items," Applied Mathematics and Computation, vol. 229, pp. 139-150, 2014.

[27] R. J. Tersine, Principle of Inventory and Material Management, North Holland, New York, NY, USA, 1994.

[28] S. S. Sana, "A production-inventory model of imperfect quality products in a three-layer supply chain," Decision Support Systems, vol. 50, no. 2, pp. 539-547, 2011. 


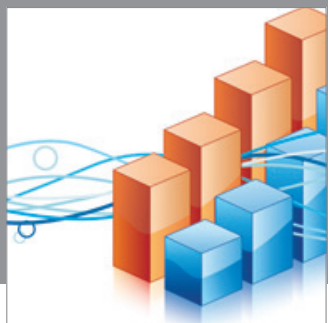

Advances in

Operations Research

mansans

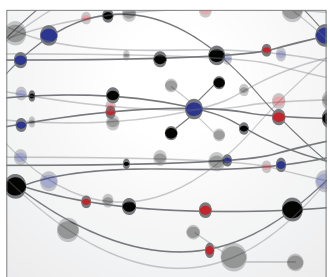

The Scientific World Journal
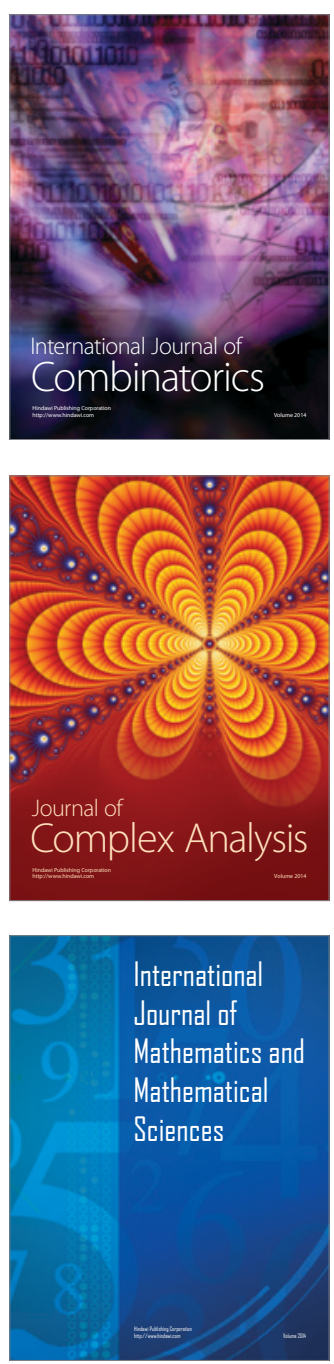
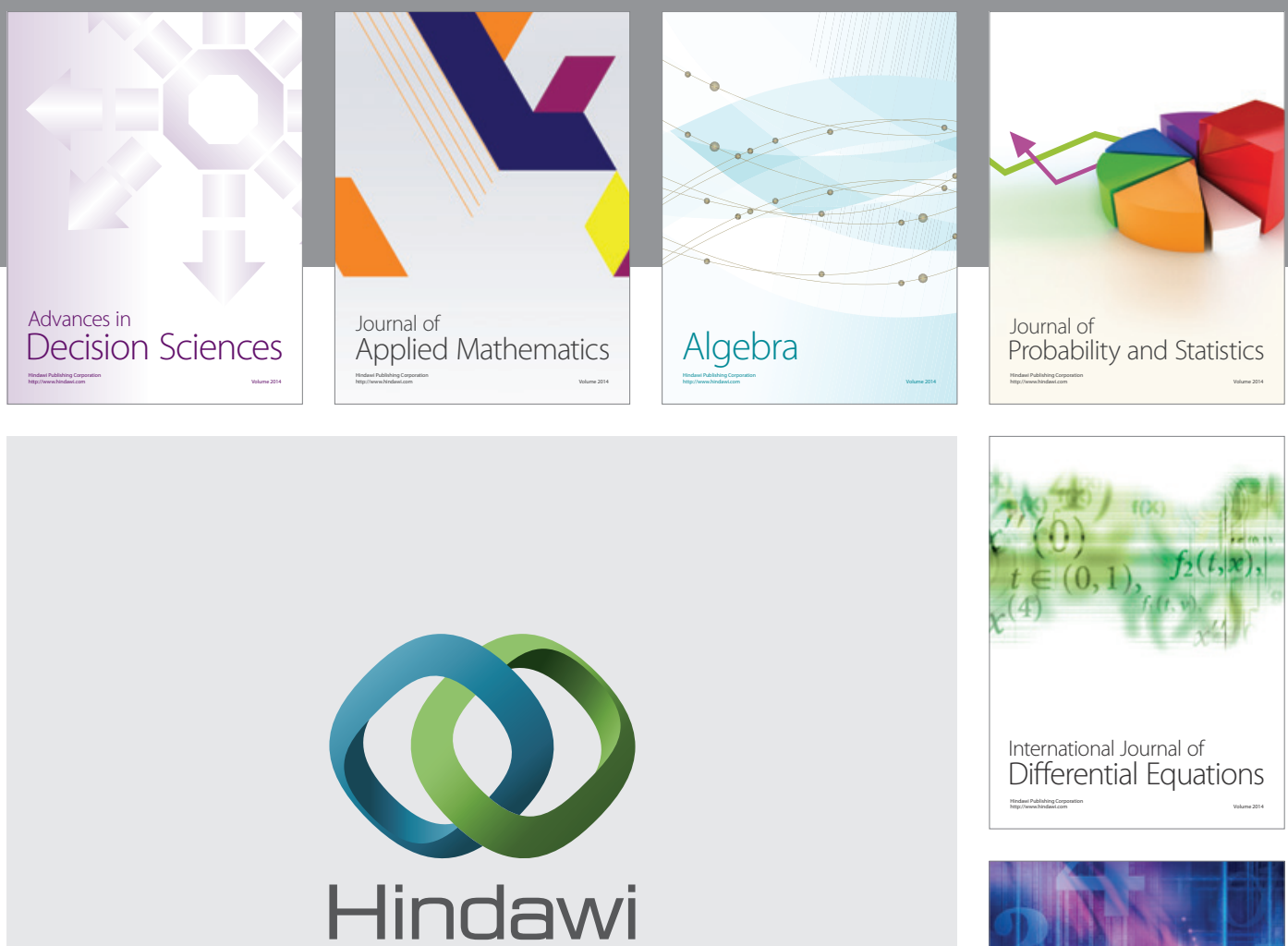

Submit your manuscripts at http://www.hindawi.com
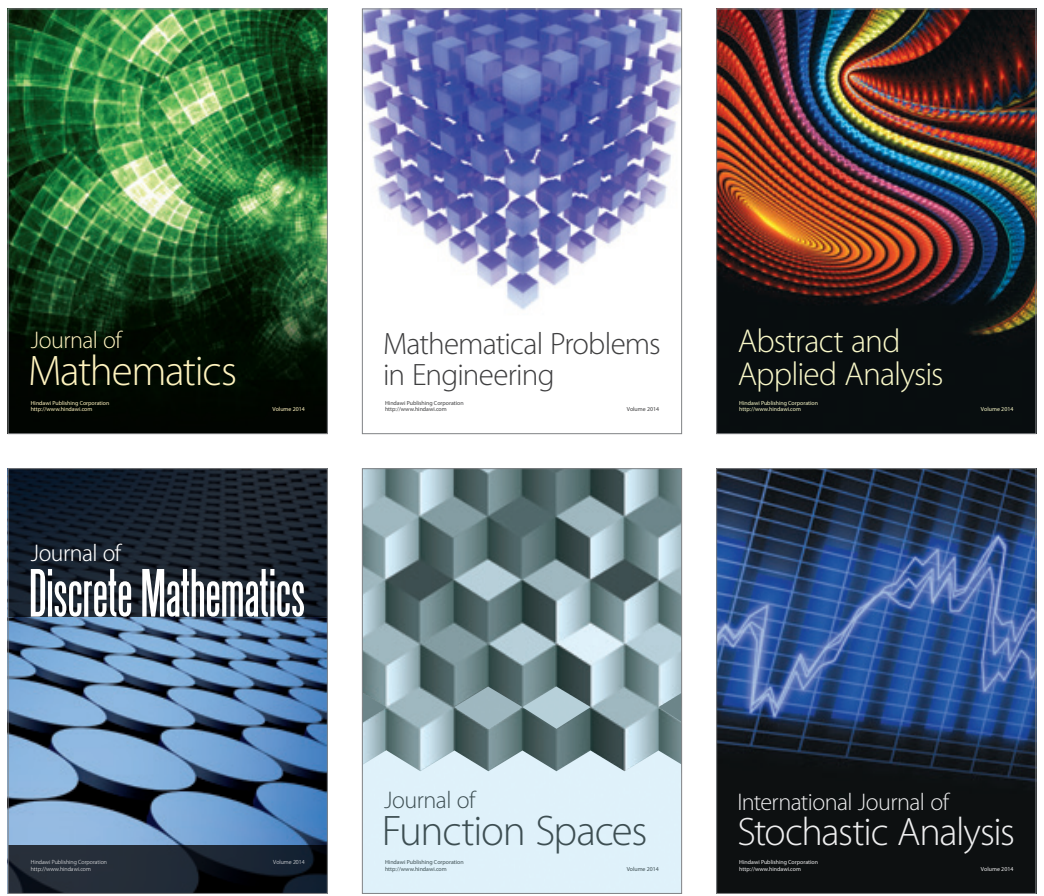

Journal of

Function Spaces

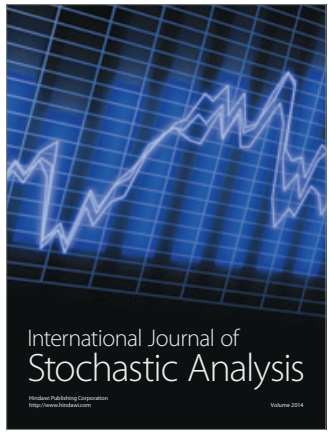

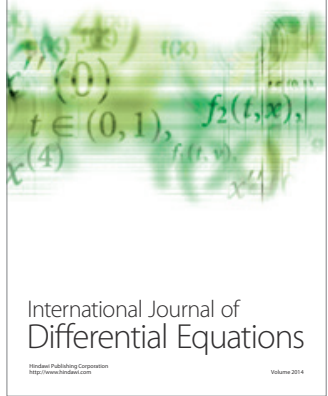
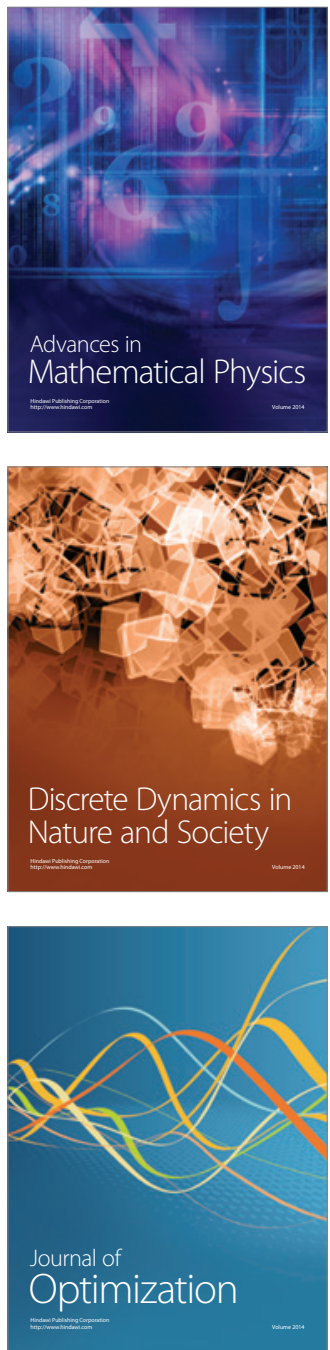